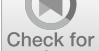

updates

Cite as

Nano-Micro Lett.

(2022) $14: 19$

Received: 14 July 2021

Accepted: 10 October 2021

Published online: 7 December 2021

(C) The Author(s) 2021

\section{Layered Foam/Film Polymer Nanocomposites with Highly Efficient EMI Shielding Properties and Ultralow Reflection}

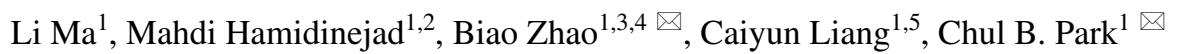

\title{
HIGHLIGHTS
}

- The successful fabrication of layered foam/film structure via the crystal melting temperature mismatch for two grades of PVDF resin in a batch foaming process.

- Developing the heterostructure interfaces between $\mathrm{SiC}$ nanowires and MXene $\left(\mathrm{Ti}_{3} \mathrm{C}_{2} \mathrm{~T}_{\mathrm{x}}\right)$ nanosheets.

- Achieving efficient electromagnetic interference shielding effectiveness with ultralow reflectivity.

ABSTRACT Lightweight, high-efficiency and low reflection electromagnetic interference (EMI) shielding polymer composites are greatly desired for addressing the challenge of ever-increasing electromagnetic pollution. Lightweight layered foam/film PVDF nanocomposites with efficient EMI shielding effectiveness and ultralow reflection power were fabricated by physical foaming. The unique layered foam/film structure was composed of PVDF/SiCnw/MXene $\left(\mathrm{Ti}_{3} \mathrm{C}_{2} \mathrm{~T}_{\mathrm{x}}\right)$ composite foam as absorption layer and highly conductive PVDF/MWCNT/ GnPs composite film as a reflection layer. The foam layer with numerous heterogeneous interfaces developed between the SiC nanowires ( $\mathrm{SiCnw}$ ) and 2D MXene nanosheets imparted superior EM wave attenuation capability. Furthermore, the microcellular structure effectively tuned the impedance matching and prolonged the wave propagating path by internal scattering and multiple reflections. Meanwhile, the highly conductive PVDF/MWCNT/GnPs composite ( 220 $\mathrm{S} \mathrm{m}^{-1}$ ) exhibited superior reflectivity $(\mathrm{R})$ of 0.95 . The tailored structure in the

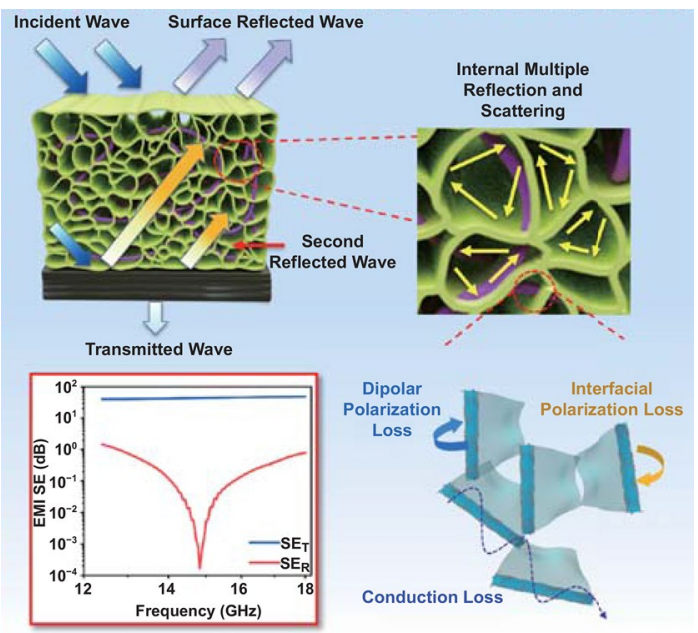
layered foam/film PVDF nanocomposite exhibited an EMI SE of $32.6 \mathrm{~dB}$ and a low reflection bandwidth of $4 \mathrm{GHz}(\mathrm{R}<0.1)$ over the Kuband $(12.4-18.0 \mathrm{GHz})$ at a thickness of $1.95 \mathrm{~mm}$. A peak $\mathrm{SE}_{\mathrm{R}}$ of $3.1 \times 10^{-4} \mathrm{~dB}$ was obtained which corresponds to only $0.0022 \%$ reflection

Biao Zhao,biao_zhao@zua.edu.cn; Chul B.Park, park@mie.utoronto.ca

1 Department of Mechanical and Industrial Engineering, University of Toronto, 5 King's College Road, Toronto, ON M5S 3G8, Canada

2 Institute for Manufacturing, Department of Engineering, University of Cambridge, Cambridge CB3 OFS, UK

3 Laboratory of Advanced Materials, Department of Materials Science, Collaborative Innovation Center of Chemistry for Energy Materials, Fudan University, Shanghai 200438, People's Republic of China

4 Henan Key Laboratory of Aeronautical Materials and Application Technology, School of Material Science and Engineering, Zhengzhou University of Aeronautics, Zhengzhou, Henan 450046, People's Republic of China

5 CAS Key Laboratory of High-Performance Synthetic Rubber and Its Composite Materials, Changchun Institute of Applied Chemistry, Chinese Academy of Sciences, Changchun 130022, People's Republic of China 
efficiency. In consequence, this study introduces a feasible approach to develop lightweight, high-efficiency EMI shielding materials with ultralow reflection for emerging applications.

KEYWORDS 2D MXene nanosheets; SiC nanowires; Layered foam/film polymer nanocomposites; Microcellular structure;

Absorption-dominated EMI shielding

\section{Introduction}

The rapid development of fifth-generation (5G) wireless systems, satellite communications and portable electronic devices is contributing to intensified electromagnetic wave emissions to the environment, which results in severe electromagnetic pollution [1-3]. The electromagnetic interference (EMI) can lead to malfunction of sensitive electronic components and system failure and may endanger human health [4-7]. To address the concerns caused by EMI pollution, substantial efforts have been devoted to developing high-efficiency EM wave attenuation and EMI shielding materials [8-12].

Efficient EMI shielding can be achieved by materials with high electrical conductivity [13]. For instance, 2D transition metal carbides (MXene) have recently been reported as promising EMI shielding materials owing to their superior electrical conductivity $\left(\sim 4500 \mathrm{~S} \mathrm{~cm}^{-1}\right)$ [14]. Shahzad et al. [15] demonstrated an ultraefficient EMI shielding effectiveness (SE) of $92 \mathrm{~dB}$ for a freestanding film of $\mathrm{Ti}_{3} \mathrm{C}_{2} \mathrm{~T}_{\mathrm{x}}$ MXene at a thickness of $45 \mu \mathrm{m}$. Iqbal et al. [16] demonstrated that the shielding performance of freestanding MXene films could be further enhanced through heat treatment; for instance, heat-treated $\mathrm{Ti}_{3} \mathrm{CNT}_{\mathrm{x}}$ MXene films offered an EMI $\mathrm{SE}$ of $116 \mathrm{~dB}$ at a thickness of $40 \mu \mathrm{m}$. Incorporating highly conductive nanomaterials such as MXene nanosheets in manufacturing polymer composites has shown great promise as efficient EMI shielding materials. Polymer composites have an attractive array of properties such as light weight, ease of processing, chemical stability, low cost and adjustable electrical and thermal properties [17-20]. The heterostructured interfaces, such as $\mathrm{GnPs} / \mathrm{SiC}$ [21], MXene $/ \mathrm{Ni}$ [22], SiC/C [23], NiCo@ C/ZnO [24], C/MOS 2 [25], Fe/ $\mathrm{MnO} @ \mathrm{C}$ [26] and CNTs/Fe [27], constructed in the composite matrix can be a promising way to promote the EM wave dissipation capability by dielectric loss and/or magnetic loss and eventually convert the energy into heat.

In spite of the interesting properties of conductive polymer composites with outstanding EMI SE [28, 29], the severe impedance mismatch between solid and air results in significant reflection of the incident EM wave, leading to secondary EMI pollution in the environment. For instance, Song et al. [30] reported a highly conductive PDMS/cellulose carbon aerogel/rGO composite $\left(75 \mathrm{~S} \mathrm{~m}^{-1}\right)$ which exhibited an optimized EMI SE of $44 \mathrm{~dB}$ with $\mathrm{SE}_{\mathrm{R}}$ of $7 \mathrm{~dB}$. This means $99.996 \%$ of the incident EM waves are shielded, but $80 \%$ of waves are reflected at the surface. Therefore, absorption-dominated EMI shielding materials with high EM wave dissipation capability are in great demand [31-33].

Conductive polymer composite foams with microcellular structure can effectively tune the impedance matching and enhance the attenuation of EM waves via internal scattering and multiple reflections within the cellular structure [17, 19, 34-39]. Zhao et al. [17] demonstrated absorption-dominated EMI shielding properties for microcellular PVDF/10 wt \% GnPs composite foams. Benefiting from the unique cellular structure, the composite foam exhibited an EMI SE of $27 \mathrm{~dB}$ with a void fraction (VF) of $48 \%$, and the corresponding reflection effectiveness $\mathrm{SE}_{\mathrm{R}}$ was only $2.6 \mathrm{~dB}$, which indicated a reflection efficiency of $46 \%$ for the incident EM wave. To develop shielding materials with ultralow reflection, the surface impedance matching should be carefully tuned by increasing the degree of foaming and decreasing the effective dielectric permittivity [40, 41]. However, such materials can hardly obtain efficient EMI SE due to insufficient electrical conductivity and dissipation capability [42-44]. Zhao et al. [42] demonstrated an optimized $\mathrm{SE}_{\mathrm{R}}$ of $1.6 \mathrm{~dB}$ but insufficient EMI SE of $12.8 \mathrm{~dB}$ by increasing the VF of composite foam from 36 to $60 \%$. It is still challenging to fabricate efficient EMI shielding material with ultralow reflectivity. To address this drawback, Duan et al. [45] introduced a graded conductive polymer composite foam via freeze drying. Such gradient structures can achieve both low reflectivity and high EMI SE by combining (i) the absorption layer, with excellent impedance matching and high attenuation capability, and (ii) the reflection layer with superior electrical conductivity and high reflection capability. A waterborne polyurethane/FeCo@rGO/Ag-coated expanded polymer bead (EBAg) composite foam exhibited an average EMI SE of $84 \mathrm{~dB}$ with an average $\mathrm{SE}_{\mathrm{R}}$ of $0.32 \mathrm{~dB}$ 
$[46,47]$. Despite the importance of shielding materials with ultralow reflection, the field is at an early stage, and there are very few research works devoted to the manufacture of efficient ultralow reflection shielding materials.

In this work, a novel design of lightweight, layered foam/film PVDF nanocomposite with efficient EMI shielding effectiveness and ultralow reflection characteristics is reported. The foam/film composites were fabricated based on the mismatched crystal melting temperatures of two grades of PVDF resin from the batch foaming process. The layered foam/film nanocomposites were composed of an upper PVDF/SiCnw@MXene composite foam as impedance matching and wave attenuation layer, and a bottom highly conductive PVDF/MWCNT/Graphene nanoplatelets (GnPs) composite film as the reflection layer. A percolated MWCNT/GnPs network constructed in the PVDF matrix resulted in an efficient EMI SE (33.49 dB) with high reflectivity due to superior electrical conductivity $\left(\sim 220 \mathrm{~S} \mathrm{~m}^{-1}\right)$ and high impedance mismatch. The numerous heterogeneous interfaces developed between SiCnw and MXene nanosheets in the PVDF matrix enhanced the EM wave attenuation capability by interfacial polarization loss and conduction loss. Meanwhile, the incorporation of microcellular structure can effectively tune the surface impedance matching and internal scattering. The degree of foaming and composition of SiCnw/MXene hybrids were optimized to maximize low reflection bandwidth (reflection less than 10\%) and minimize the reflection efficiency over the Ku-band $(12.4-18.0 \mathrm{GHz})$. As a result, this study introduces a simple method to develop lightweight, efficient low reflection EMI shielding materials.

\section{Materials and Methods}

\subsection{Materials}

Commercial grade polyvinylidene fluoride-co-hexafluoropropylene (PVDF-HFP), Kynar2800, and polyvinylidene fluoride (PVDF), Kynar740, with specific gravity of 1.78 and $1.8 \mathrm{~g} \mathrm{~cm}^{-3}$, respectively, were kindly donated by ArkemaNA. $\mathrm{SiC}$ nanowires (SiCnw), with a diameter of 50-600 nm, were purchased from Nanjing XFNano Materials Tech Co., Ltd. Graphene nanoplatelet (GnP) powder was purchased from SuperiC Technology Ltd. (China). Carbon nanotube (CNT) powder, NC7000t, was obtained from Nanocyl SA, Belgium. Hydrochloric acid ACS reagent Grade (12 Normal) was purchased from BioShop Canada Inc. Lithium fluoride (LiF) powder ( $\geq 99.9 \%), \mathrm{N}, \mathrm{N}$-dimethylformamide (DMF) and poly (diallyl dimethylammonium chloride) (PDDA) were purchased from Sigma-Aldrich. $\mathrm{Ti}_{3} \mathrm{AlC}_{2}$ powder (400 mesh) was purchased from 11 Technology Co., Ltd. (China).

\subsection{Preparation of PVDF-HFP/SiCnw@MXene Nanocomposites}

The synthesis process of few-layer $\mathrm{Ti}_{3} \mathrm{C}_{2} \mathrm{~T}_{\mathrm{x}}$ MXene and modified SiCnw was introduced in previous work, and more details can be seen in ESI [48]. The schematic illustration of fabrication is shown in Fig. 1. Firstly, $0.07 \mathrm{~g}$ of $\mathrm{Ti}_{3} \mathrm{C}_{2} \mathrm{~T}_{\mathrm{x}}$ MXene was dispersed in $30 \mathrm{~mL}$ of DMF followed by $15 \mathrm{~min}$ sonication in an Ar environment. Then, $0.49 \mathrm{~g}$ of the modified SiCnw was added to the MXene/DMF solution and sonicated for another $15 \mathrm{~min}$. The SiCnw/MXene was then uniformly dispersed in DMF solution under continuous stirring for $30 \mathrm{~min}$. The modified SiCnw and MXene 2D sheets can be assembled together, driven by electrostatic force during the process. After that, $1.2 \mathrm{~g}$ of PVDF-HFP was dissolved and dispersed in the mixture via continuous stirring for $3 \mathrm{~h}$ at $70{ }^{\circ} \mathrm{C}$. The resulting PVDF-HFP/SiCnw @ MXene/DMF solution was then injected into a water tank with magnetic stirring at the bottom via a syringe for quick phase inversion. Finally, after drying in a vacuum oven at $70{ }^{\circ} \mathrm{C}$ overnight, the product was hot-pressed at $200{ }^{\circ} \mathrm{C}$ to obtain PVDF/30 wt $\%$ SiCnw@MXene nanocomposites $\left(1.48 \times 15.8 \times 20 \mathrm{~mm}^{3}\right)$. Different compositions of SiCnw and MXene hybrids were applied: SiCnw@MXene 5:1 (0.467 and 0.093 g), SiCnw@ MXene 7:1 (0.49 and 0.07 g), SiCnw@MXene 9:1 (0.504 and $0.056 \mathrm{~g})$ and neat $\mathrm{SiCnw}(0.56$ and $0 \mathrm{~g})$.

\subsection{Preparation of PVDF/GnPs/MWCNT Nanocomposites}

Firstly, $0.1 \mathrm{~g}$ of GnPs and $0.1 \mathrm{~g}$ of MWCNTs were dispersed in $25 \mathrm{~mL}$ of DMF and sonicated for $30 \mathrm{~min}$. Then, $0.8 \mathrm{~g}$ of PVDF was dissolved and dispersed in the solution via continuous stirring for $3 \mathrm{~h}$ at $70{ }^{\circ} \mathrm{C}$. After mixing, the same quick phase inversion technique was applied. Finally, the product was hot-pressed at $200{ }^{\circ} \mathrm{C}$ to obtain $\mathrm{PVDF} / 10 \mathrm{wt} \%$ GnPs/10 wt $\%$ MWCNT nanocomposite $(0.4 \times 15.8 \times 20$ $\left.\mathrm{mm}^{3}\right)$. 


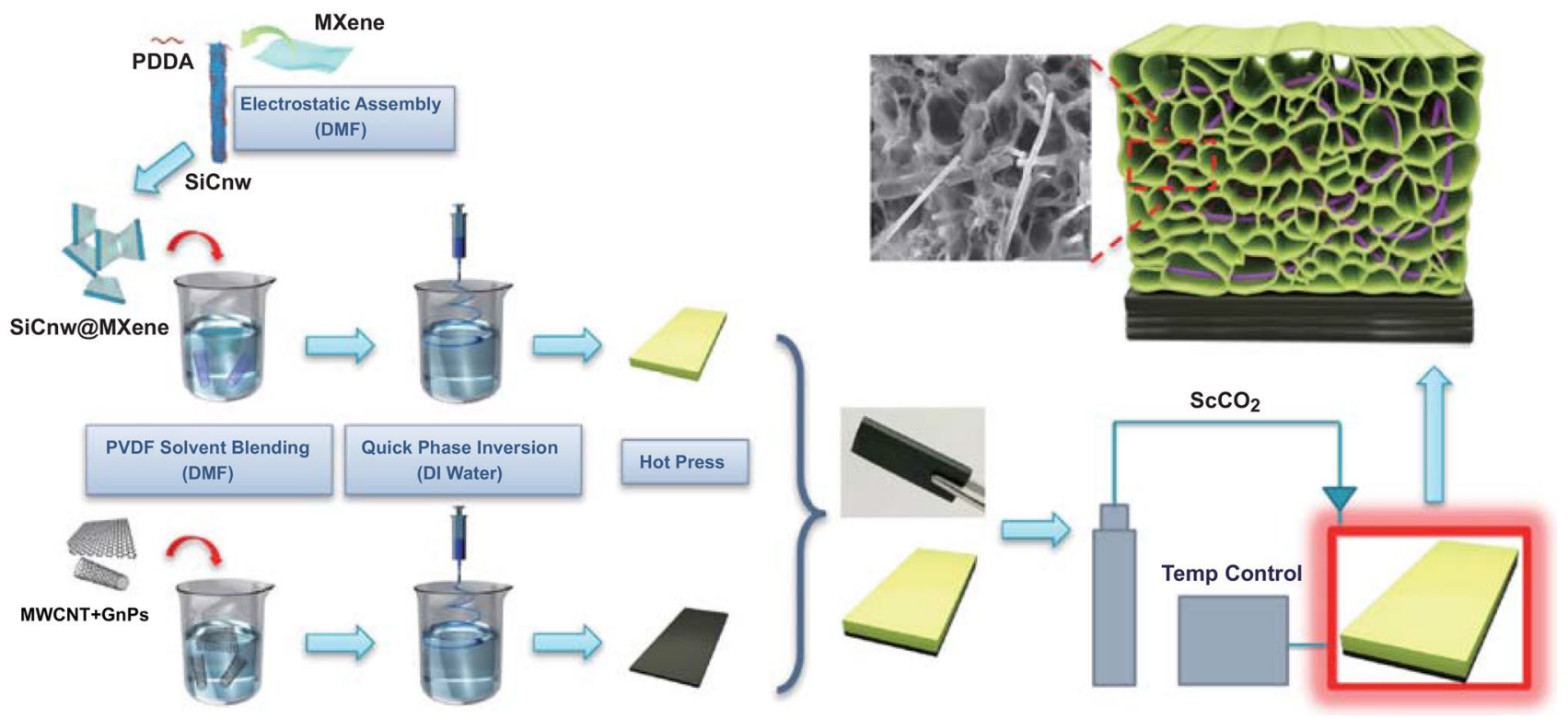

Fig. 1 Schematic illustration of layered foam/film PVDF nanocomposite fabrication

\subsection{Fabrication of Layered Foam/Film PVDF Nanocomposites}

As shown in Fig. 1, 1.8 mm of PVDF/SiCnw@MXene composite and $0.4 \mathrm{~mm}$ of PVDF/GnPs/MWCNT composite were layered and placed in a mold $\left(2.2 \times 15.8 \times 20 \mathrm{~mm}^{3}\right)$, and the two composites were hot-pressed together at $200{ }^{\circ} \mathrm{C}$. Then, the double layer composites obtained were placed in a high-pressure batch foaming chamber, filled with 2000 psi supercritical carbon dioxide, and saturated at various temperatures for $30 \mathrm{~min}$. The saturation temperatures were set below the onset temperature of PVDF crystal melting $\left(163^{\circ} \mathrm{C}\right)$ to avoid foaming the film layer, while the PVDF-HFP layer could still be foamed. After saturation, the gas was quickly released and the chamber quenched in an ice bath to obtain the layered foam/ film PVDF nanocomposites, where PVDF-HFP/SiCnw@ MXene foam sits on PVDF/GnPs/MWCNT film. The influence of saturation temperature $\left(128\right.$ to $\left.143^{\circ} \mathrm{C}\right)$ on the VF of foamed layer was investigated, as shown in Table S1. In this study, PVDF-HFP/SiCnw@MXene foams with VF around
$45 \%, 55 \%$ and $65 \%$ were selected to study the effect of VF on their EMI shielding performance.

\subsection{Characterization}

The samples' broadband alternating current (AC) conductivity was tested by a KEYSIGHT impedance analyzer (E4990A, $20 \mathrm{~Hz}$ to $10 \mathrm{MHz}$ ). Field emission scanning electron microscopy (SEM, FEI Quanta FEG 250, with EDX) and transmission electron microscopy (TEM, Tecani G2) were applied to investigate and observe microstructure and morphology of the samples. An X-ray diffractometer (XRD, Rigaku MiniFlex 600) using $\mathrm{Cu}-\mathrm{Ka}$ as a radiation resource was applied to investigate the crystal structure of samples. The elemental chemical bonding in MXene was confirmed by X-ray photoelectron spectroscopy (XPS, Thermo Scientific Theta Probe) using Al-Ka as a radiation resource. The temperature-dependent storage modulus of the samples was investigated by the Dynamic mechanical analysis (DMA) instrument (TA DMA q800, tensile fixture, 30-80 ${ }^{\circ} \mathrm{C}$, oscillation frequency $1 \mathrm{~Hz}$, oscillation strain $0.02 \%$ ). 


\subsection{Measurement of Electromagnetic Wave Interference Shielding Effectiveness}

The $\mathrm{S}$ parameters $\left(\mathrm{S}_{11}\right.$ and $\left.\mathrm{S}_{21}\right)$ of each sample were collected by using the waveguide mode in the frequency range 12.4-18.0 GHz (Ku-band) on a vector network analyzer (VNA, KEYSIGHT, PNA-L Series N5234B). Samples were cut and trimmed into a $15.8 \times 7.9 \mathrm{~mm}^{2}$ rectangular shape to precisely fit the waveguide fixture. The reflection shielding effectiveness $\left(\mathrm{SE}_{\mathrm{R}}\right)$ and the total shielding effectiveness $\left(\mathrm{SE}_{\mathrm{T}}\right)$ can be determined by the following equations using $S_{11}$ and $S_{21}$ parameters [21,37]:

$R=\left|S_{11}^{2}\right|, T=\left|S_{21}^{2}\right|, A+T+R=1$

$\mathrm{SE}_{\mathrm{R}}=-10 \log (1-\mathrm{R})$

$\mathrm{SE}_{\mathrm{T}}=-10 \log (\mathrm{T})$

where $\mathrm{A}, \mathrm{T}$ and $\mathrm{R}$ are the absorptivity, transmissivity and reflectivity for the incident EM wave, respectively.
Moreover, the absorption effectiveness of the incident wave can be described as following [17, 49]:

$\mathrm{SE}_{A}=-10 \log \left(1-\frac{A}{1-R}\right)=\mathrm{SE}_{T}-\mathrm{SE}_{R}-\mathrm{SE}_{M}$

when the $\mathrm{SE}_{T}$ is larger than $15 \mathrm{~dB}$, multiple reflections $\left(\mathrm{SE}_{\mathrm{M}}\right)$ are generally negligible. The influence of sample thickness on EMI shielding performance was investigated by trimming the sample thickness using sandpaper.

\section{Results and Discussion}

\subsection{Microstructure and Morphology}

Driven by the electrostatic force, the flexible $2 \mathrm{D} \mathrm{Ti}_{3} \mathrm{C}_{2} \mathrm{~T}_{\mathrm{x}}$ nanosheets can wrap over the surface of modified 1D SiCnw [48, 50], and the microstructure and morphology of such a heterostructure are illustrated in Figs. 2 and S1. TheSiCnw@MXene heterostructure can also be observed in PVDF /SiCnw@MXene composite foam. As shown in Figs. 2c, f and S1, the EDX-SEM image confirms that the
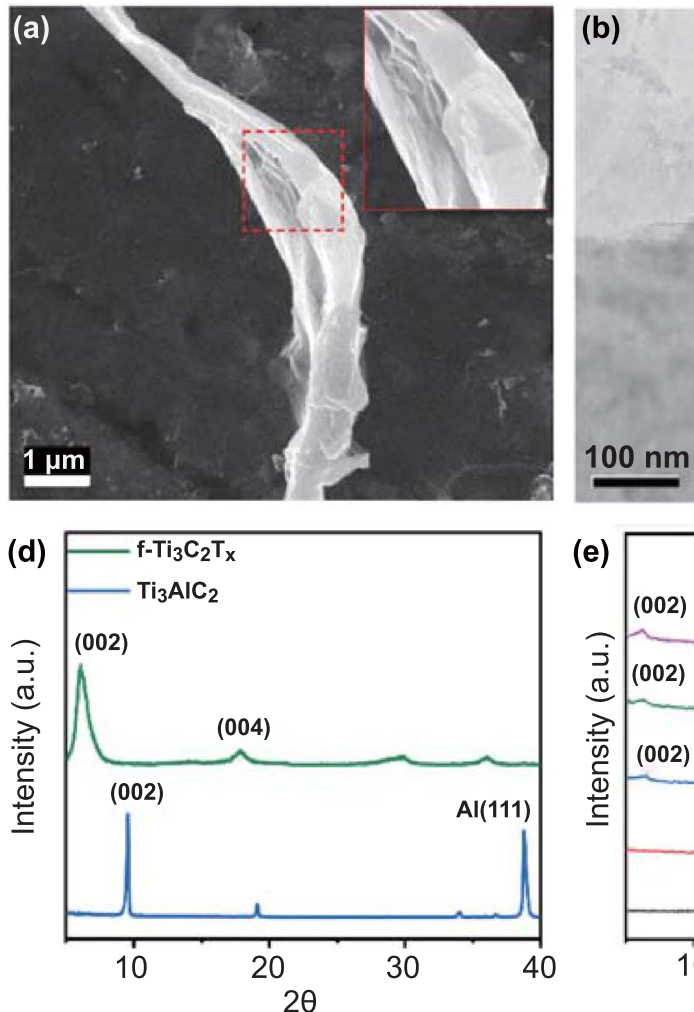

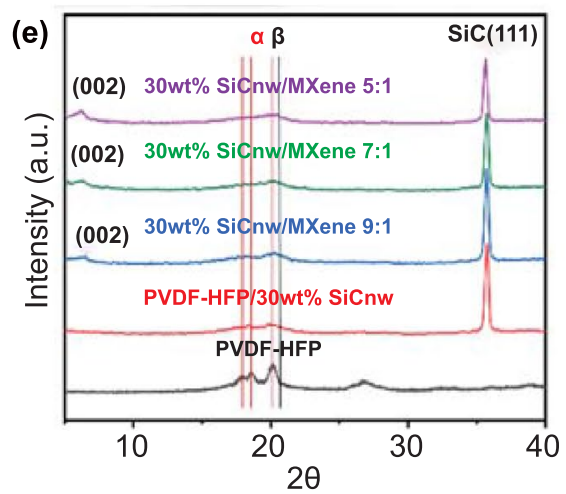

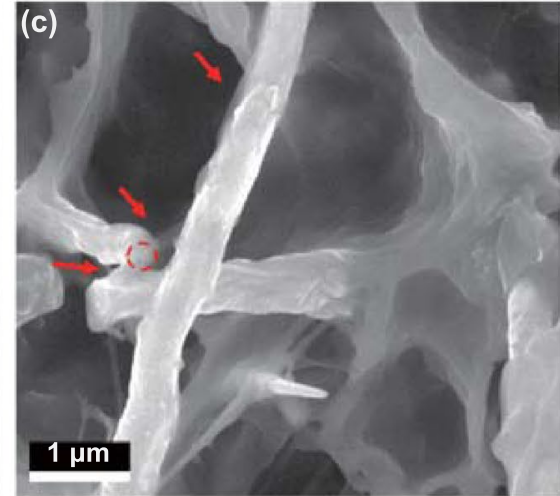

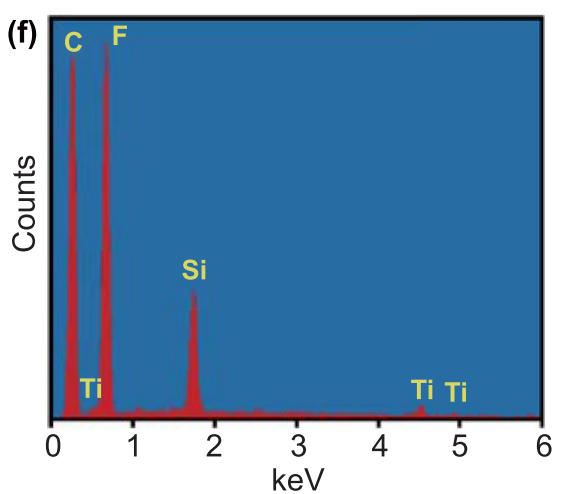

Fig. 2 a SEM image of self-assembled $\mathrm{SiCnw} @ \mathrm{Ti}_{3} \mathrm{C}_{2} \mathrm{~T}_{\mathrm{x}}$ nanostructure; $\mathbf{b} \mathrm{TEM}$ image of $\mathrm{SiCnw} @ \mathrm{f}-\mathrm{Ti} \mathrm{C}_{2} \mathrm{~T}_{\mathrm{x}}$; $\mathbf{c} \mathrm{EDX}-\mathrm{SEM}$ image of PVDFHFP/SiCnw@MXene composite foam (30 wt \%, 45\% VF); d XRD patterns of $\mathrm{Ti}_{3} \mathrm{AlC}_{2}, \mathrm{Ti}_{3} \mathrm{C}_{2} \mathrm{~T}_{\mathrm{x}}$; e XRD patterns of PVDF-HFP composites; $\mathbf{f}$ EDX pattern of the red circle in c 
SiCnw@MXene heterostructure is preserved after solvent casting, hot pressing and foaming.

The phase and crystal structure of as-prepared MXene film and fabricated nanocomposites were examined using XRD analysis. Also, after selective etching, the peak corresponding to the (002) crystal planes of $\mathrm{Ti}_{3} \mathrm{AlC}_{2}$ shifted from $\sim 9.7^{\circ}$ to $\sim 6.6^{\circ}$ because of an increase in the d-spacing of the $\mathrm{Ti}_{3} \mathrm{C}_{2} \mathrm{~T}_{\mathrm{x}}$ (Fig. 2d) $[15,51,52]$. The absence of the (111) plane peak of $\mathrm{Al}$ in X-Ray spectra of the few-layer $\mathrm{Ti}_{3} \mathrm{C}_{2} \mathrm{~T}_{\mathrm{x}}$ nanosheets is an indication of successful manufacture of MXene nanosheets. The XRD pattern of PVDF/ SiCnw@MXene composites with various filler compositions is shown in Fig. 2e; the peak at $\sim 35.7^{\circ}$ corresponds to the (111) plane of $\mathrm{SiC}[23,53,54]$. The intensity of the (002) plane of MXene increases with MXene content in the composite. Also, the XRD pattern of the PVDF/SiCnw @ MXene composites illustrates that the incorporation of $\mathrm{Ti}_{3} \mathrm{C}_{2} \mathrm{~T}_{\mathrm{x}}$ nanosheets and SiCnw could promote the formation of PVDF $\beta$ crystals. The promoted $\beta$ phase can be attributed to the extensional stress around the embedded filler in the PVDF melt [55], which can help to align polymer chains and to form the electroactive $\beta$ phase crystal [56].

Electronic states of the Ti element within $\mathrm{Ti}_{3} \mathrm{C}_{2} \mathrm{~T}_{\mathrm{x}}$ MXene nanosheets can be found in Fig. S2. The fabrication of PVDF/MWCNT/GnPs nanocomposite is supported by the XRD spectra in Fig. S3. The characteristic peak of MWCNT and $\mathrm{GnPs}$ was detected around $26^{\circ}$.

Figure 3a shows the cross-sectional SEM images of layered foam/film PVDF nanocomposites (PVDF/SiCnw @ MXene composite foam and PVDF/MWCNT/GnPs composite film). The microstructure of the layered foam/film PVDF nanocomposites before foaming is shown in Fig. S4d, e. The microcellular morphology in Fig. 3a confirms the successful foaming of the upper PVDF composite. An appropriate foaming temperature window (128 to $162{ }^{\circ} \mathrm{C}$ ) of the PVDF composites guarantees the formation of microcellular structure in the upper layer and keeping the bottom layer solid, as shown in Figs. 3a and S4a, b. The endothermal plots for two grades of PVDF are presented in Fig. S5. Therefore, the existing crystal lamellae in the PVDF matrix will be the gas barrier and prevent gas molecules getting into the bottom high melting temperature PVDF matrix [57]. It is worth noting that foaming of the upper layer composite is constrained around the interface, and only some small cells are observed. In the meantime, a thin layer of interface stayed in solid state between two layers, which can be the indication of blending of two grades of PVDF at the interface during the hot pressing. The interface layer is visible in the solid PVDF nanocomposite, as shown in Fig. S4f.

The high magnification SEM images of upper foamed absorbers with different VF $(0 \%, \sim 45 \%$, and $\sim 65 \%)$ are shown in Fig. 3b. In the case of the samples before foaming (solid), as shown in the first column of Fig. 3b, a percolated and agglomerated SiCnw/MXene network was constructed in the upper layer via solvent casting (PVDF/ SiCnw@MXene (30wt\%, 7:1)). As shown in the second and third columns of Fig. 3b, by inducing foaming, the cells nucleate and form the interface between the filler and polymer matrix.

According to the heterogeneous nucleation theory, driven by the thermal instability of the saturated gas molecules in the molten polymer after pressure drop, nucleation of cells will be favored at the interface between the filler and polymer matrix due to reduction in activation Gibbs free energy [39, 55, 58]. Also, the filler particles could be reoriented and realigned around the growing bubble [38, 43, 59].

It can be seen in the EDX mapping of Si element (Fig. 3b) that cells are surrounded by a percolative and distributed SiCnw network. The increase in the VF from $~ 45 \%$ to $~ 65 \%$, as shown in the second row of Fig. 3b, increased the average cell size from 3.08 to $4.79 \mu \mathrm{m}$. Figure 3 illustrates that surrounding SiCnw/MXene networks were separated as the bubble grew.

The Dynamic mechanical analysis (DMA) presented in Fig. S6 confirms that the fabricated foam/film PVDF nanocomposites offer excellent stiffness as compared to the neat PVDF. As shown in Fig. S6, the incorporation of nanofillers in the PVDF resulted in a significant stiffening effect in the temperature range of $30-80{ }^{\circ} \mathrm{C}$. The storage modulus of $1.5 \mathrm{~mm}$ layered PVDF nanocomposite (30 wt\% SiCnw@MXene7:1) increased from 603 to 3,901 MPa at $30{ }^{\circ} \mathrm{C}$, as compared to neat PVDF with the same thickness. By inducing $45 \%$ and $65 \%$ void fraction into the upper absorption layer, the storage modulus of layered foam/film PVDF nanocomposites decreased to 1,885 and 1,026 MPa, respectively. 
(a)
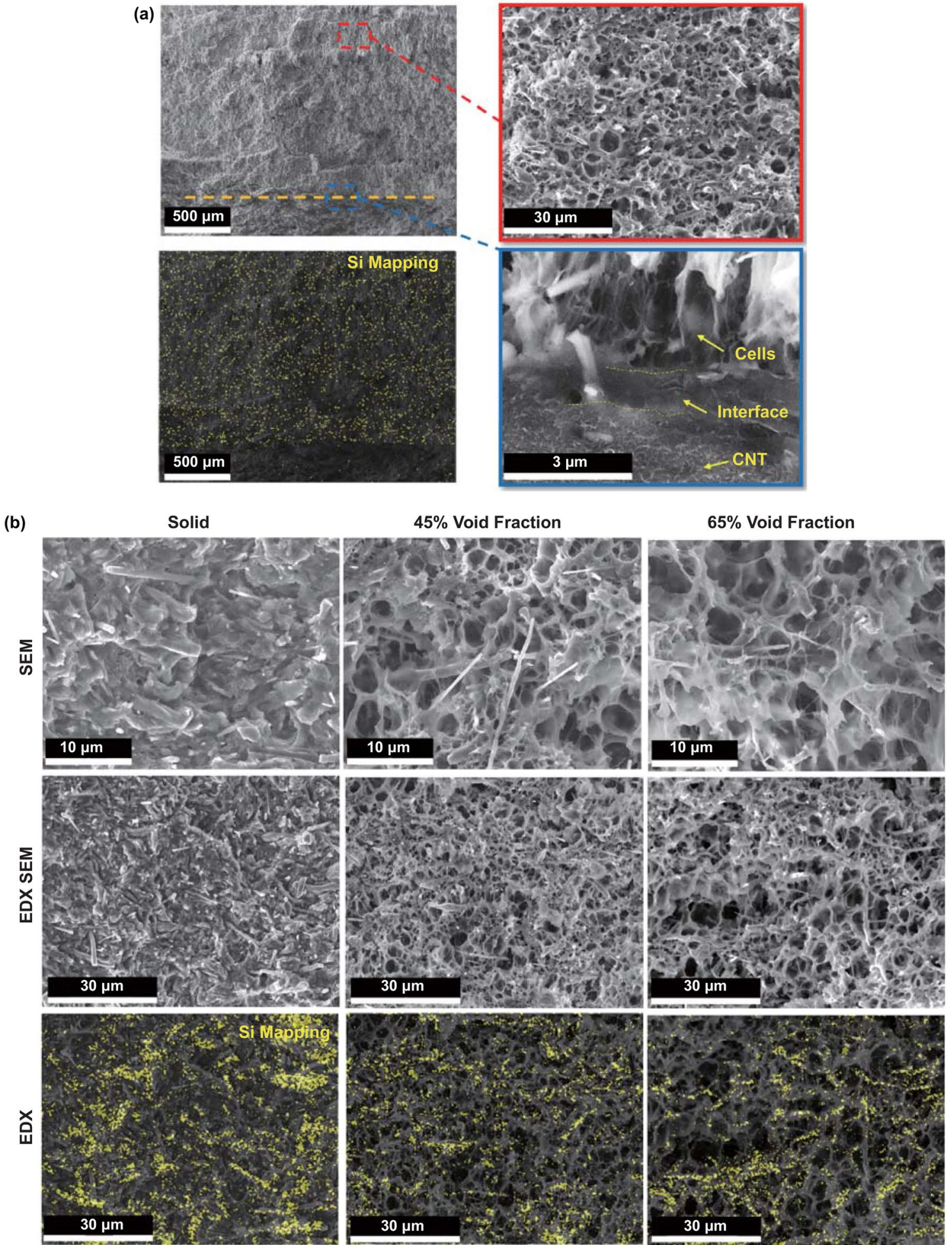

Fig. 3 Cross-sectional SEM images and Si EDX mapping of a the layered foam/film PVDF nanocomposite (upper layer VF: 45\%); b upper foamed layer with a different void fraction (30\% SiCnw@ MXene7:1, VF: 0\%, 45\% and 65\%) 


\subsection{Electrical Properties and Electromagnetic Wave Interference Shielding Performance of the Nanocomposites}

In general, the EMI shielding and impedance matching properties of EMI shielding materials are governed by electrical conductivity. Increasing the electrical conductivity can enhance the EMI SE and EM wave dissipation via conduction loss [21, 50]. However, excessively high conductivity can result in high reflectivity because of impedance mismatch [60].

To better understand the underlying mechanisms of the layered foam/film PVDF nanocomposites, the electrical and EMI performance of each layer was investigated separately. The AC conductivity for each PVDF nanocomposite with various filler compositions is shown in Fig. S7. Incorporation of MXene nanosheets in PVDF/30 wt\% SiCnw increased AC conductivity at $100 \mathrm{~Hz}$ from $4.1 \times 10^{-7}$ to $3.5 \times 10^{-6} \mathrm{~S} \mathrm{~m}^{-1}$. When the mass ratio of SiCnw to MXene decreased from 9:1 to 5:1, the addition of MXene increased the conductivity of the nanocomposites by more than two orders of magnitude at $100 \mathrm{~Hz}$. The higher conductivity of the matrix could increase the absorption of EM waves by conduction loss. On the other hand, the bottom reflection layer (PVDF/10 wt\% MWCNT/10 wt\% GnPs) exhibits frequency-independent behavior, which is an indication of a well-developed percolated conductive network in the matrix, with an AC conductivity of $220 \mathrm{~S} \mathrm{~m}^{-1}$ at $100 \mathrm{~Hz}$. The high conductivity could provide a significant impedance mismatch and lead to surface reflection of EM waves.

Dielectric loss (including dipolar and interfacial polarization loss) is another key factor governing the EM wave dissipation capability [61, 62]. According to the Maxwell-Wagner-Sillars (MWS) theory [63, 64], the development of unique heterostructures of SiCnw/MXene hybrid in the PVDF matrix can induce significant interfacial polarization loss by initiating accumulation of charge at the heterogeneous interfaces [65-67]. The EMI shielding properties of the individual layer of solid PVDF nanocomposites over the Ku-band (12.4-18 GHz) with various filler compositions are presented in Fig. 4. Incorporation of MXene nanosheets in the PVDF/30 wt\% SiCnw, slightly increased the average $\mathrm{SE}_{\mathrm{R}}$ from 3.47 to $3.75 \mathrm{~dB}$ for the PVDF/SiCnw@MXene 9:1 composite, while the average $\mathrm{SE}_{\mathrm{T}}$ was significantly
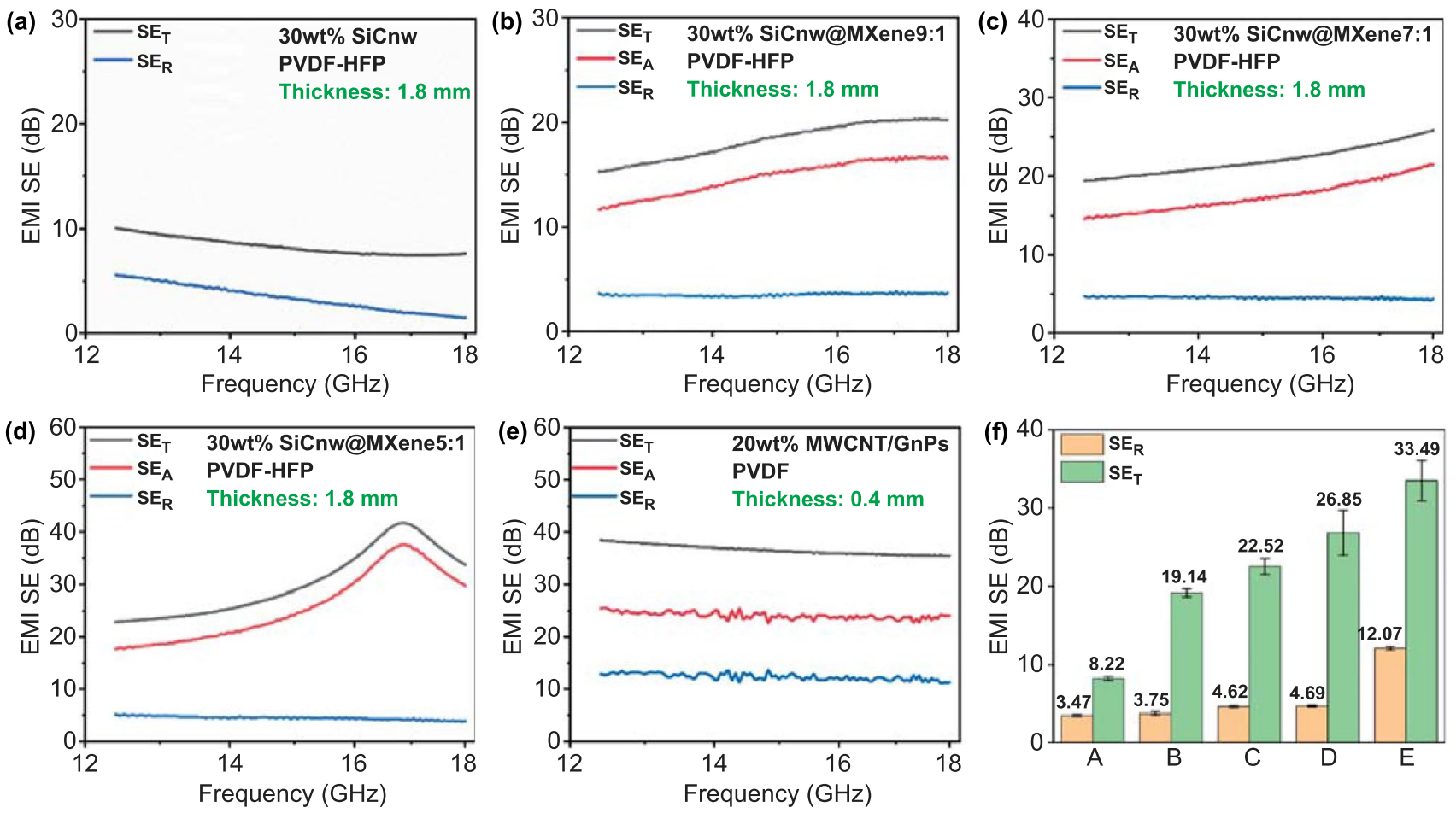

Fig. 4 a-d EMI shielding performance of PVDF/30 wt\% SiCnw@MXene nanocomposites with various filler compositions; e EMI shielding performance of PVDF/10 wt\% MWCNT/10 wt\% GnPs nanocomposite; $\mathbf{f}$ Average $\mathrm{SE}_{\mathrm{R}}$ and $\mathrm{SE}_{\mathrm{T}}$ of PVDF nanocomposites from a-e 
promoted from 8.22 to $19.14 \mathrm{~dB}$. The reflection effectiveness was almost unchanged. Thus, the enhanced EMI shielding performance is mainly attributed to the enhanced absorptivity (A). Such enhancement indicates that the incorporation of SiCnw@MXene heterostructures could significantly promote the absorption of EM waves by interfacial polarization loss [48, 68]. Meanwhile, the resonance shielding peak obtained in Fig. 5d can be attributed to the multi-reflection and scattering of propagating EM wave among the dispersedSiCnw@MXene within the matrix, which results in the enhanced absorption of incident EM wave via dielectric loss [69, 70].

Moreover, the formation of $\beta$ crystals in the PVDF matrix can help to enhance the dielectric constant by inducing dipolar polarization [71-73]. Then, based on the MWS theory, an increased dielectric constant can further enhance the interfacial polarization. Under alternating EM fields, both dipolar and interfacial polarization can turn into dielectric loss at high frequency due to relaxation processes, which then attenuate the incident EM wave. Furthermore, both $\mathrm{SE}_{\mathrm{R}}$ and $\mathrm{SE}_{\mathrm{T}}$ of PVDF/SiCnw @MXene composites gradually increased with increase in MXene content.

In previous studies, GnPs and MWCNT exhibited an excellent synergistic effect in PVDF nanocomposites, which resulted in enhanced conductivity and EMI shielding effectiveness [28]. As shown in Fig. 4e, f, the $0.4 \mathrm{~mm}$ thick reflection layer (PVDF/10 wt\% MWCNT/10 wt\% GnPs) had an average EMI SE of $33.49 \mathrm{~dB}$ and average $\mathrm{SE}_{\mathrm{R}}$ of $12.07 \mathrm{~dB}$. This indicates that more than $99.95 \%$ of the incident EM wave was shielded and $95 \%$ of the incident EM wave was reflected from the surface of the composite film.

As mentioned in the previous sections, the layered foam/ film PVDF nanocomposites were composed of PVDF/ SiCnw@MXene composite foam as an absorption layer and highly conductive PVDF/MWCNT/GnPs composite film as a reflection layer. The incident EM wave can penetrate and propagate from the upper absorption layer. To optimize the filler composition of SiCnw/MXene in the PVDF matrix as the absorber, mass ratios of SiCnw and MXene of 1:0, 9:1, 7:1, and 5:1 were investigated. The EMI SE of the layered
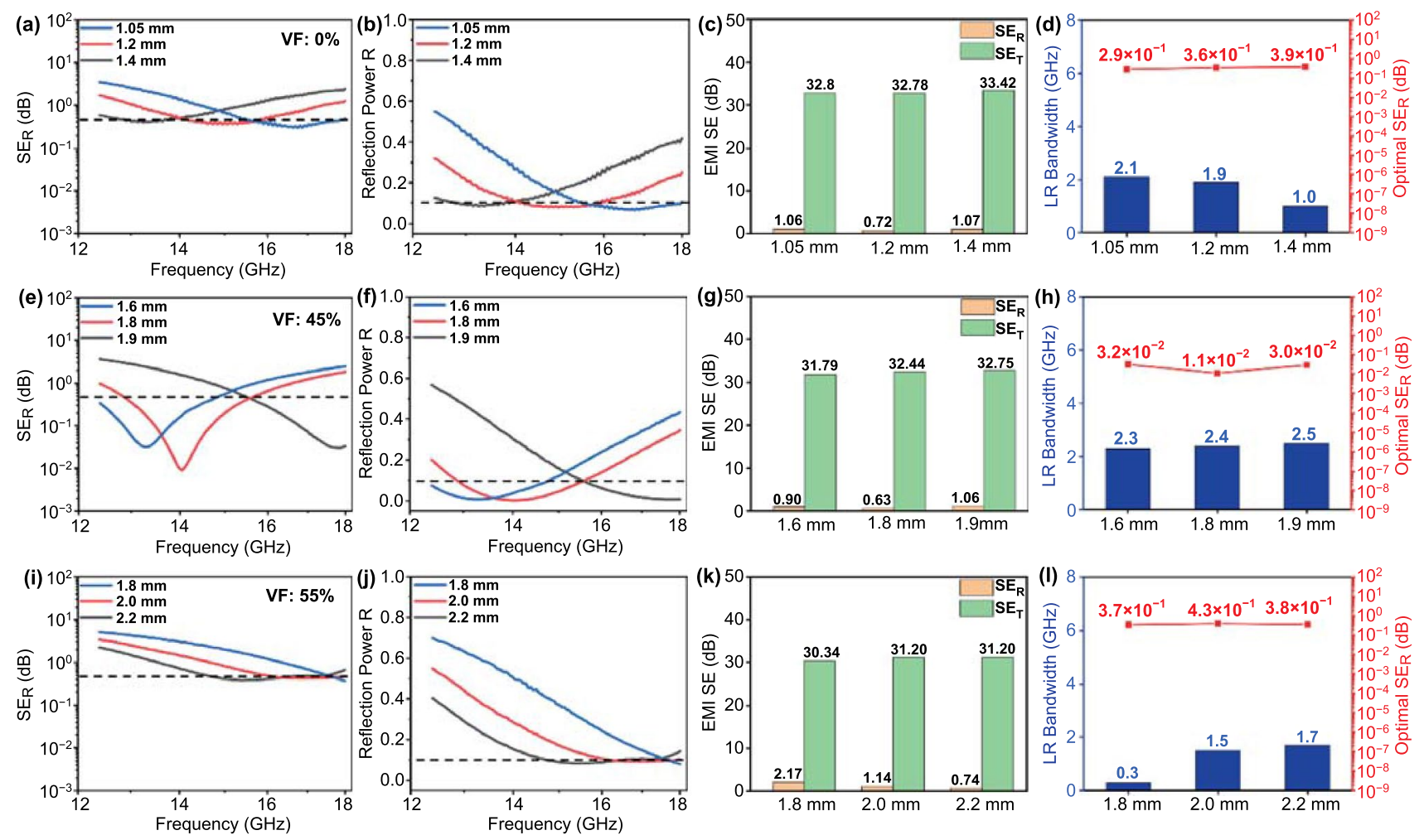
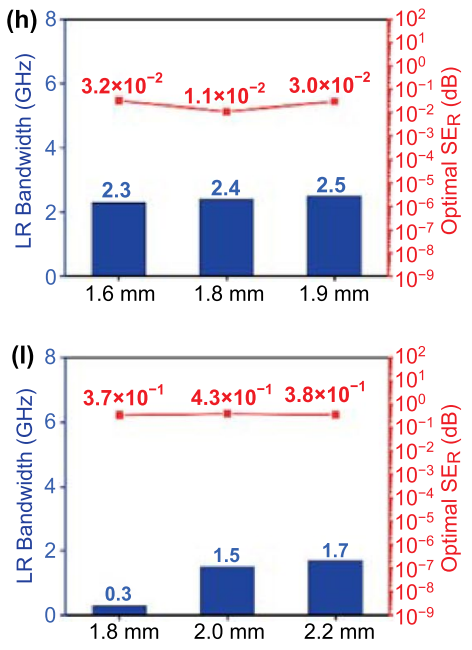

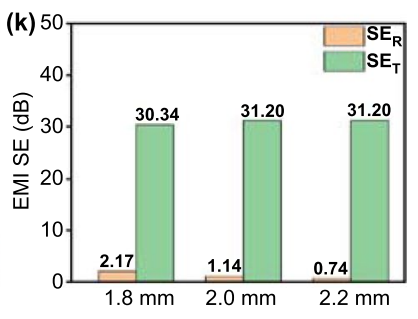

Fig. 5 EMI shielding performance for layered foam/film PVDF nanocomposites (30 wt $\% \mathrm{SiCnw}$ ) corresponding to different matching thicknesses of absorption layer; $\mathbf{a}, \mathbf{e}, \mathbf{i} \mathrm{SE}_{\mathrm{R}} ; \mathbf{b}, \mathbf{f}, \mathbf{j}$ reflectivity $\mathrm{R} ; \mathbf{c}, \mathbf{g}, \mathbf{k}$ average value of $\mathrm{SE}_{\mathrm{R}}$ and $\mathrm{SE}_{\mathrm{T}} ; \mathbf{d}, \mathbf{h}, \mathbf{l}$ low reflection bandwidth and minimal $\mathrm{SE}_{\mathrm{R}}$ value. Dependence of the VF: a-d 0\% VF; e-h 45\% VF; and i-l 55\% VF 
foam/film PVDF nanocomposites (30 wt\% SiCnw) over the $12.4-18 \mathrm{GHz}$ (Ku-band) with various VFs is presented in Fig. S8. Due to the excellent shielding performance of the reflection layer $(0.4 \mathrm{~mm} \mathrm{PVDF} / 10 \mathrm{wt} \% \mathrm{MWCNT} / 10$ $\mathrm{wt} \% \mathrm{GnPs}$ ), all nanocomposites exhibited an average $\mathrm{SE}_{\mathrm{T}}$ above $30 \mathrm{~dB}$, regardless of the thickness of the absorption layer, which means over $99.9 \%$ of incident EM wave will be shielded. Meanwhile, from Fig. 5, all the $30 \mathrm{wt} \% \mathrm{SiCnw}$ layered PVDF nanocomposites exhibited a minimum value of $\mathrm{SE}_{\mathrm{R}}$ over the Ku-band. This minimum value of $\mathrm{SE}_{\mathrm{R}}$ shifts toward higher frequency with decreasing thickness of the absorption layer. This can be explained by the law of quarter wavelength cancelation $[74,75]$ :

$t_{m}=n \frac{\lambda}{4}=n \frac{c}{4 f_{m} \sqrt{\left|\mu_{r}\right|\left|\varepsilon_{r}\right|}}(n=1,3,5 \ldots)$

where $t_{m}$ is the thickness of the absorption layer, $\lambda$ is the wavelength of EM wave, $f_{m}$ is the peak frequency with the minimal $\mathrm{SE}_{\mathrm{R}},\left|\mu_{r}\right|$ and $\left|\varepsilon_{r}\right|$ refer to the complex permeability and permittivity of the sample, and c is the speed of the light. When the thickness of the absorption layer equals a quarter of the penetrating wavelength multiplied by an odd number, the EM wave will be dissipated by interference, where the second reflection of the wave has $180^{\circ}$ phase difference relative to the surface reflection. It is worth noting that, in EM wave attenuation-related applications, the bandwidth with more than $90 \%$ absorption efficiency is defined as the effective absorption band [76, 77]. Therefore, in this work, with an average shielding efficiency of $99.95 \%$, the bandwidth with $\mathrm{SE}_{\mathrm{R}}$ less than $0.45 \mathrm{~dB}$ will be defined as the low reflection bandwidth (LR bandwidth). $\mathrm{SE}_{\mathrm{R}}$ less than $0.45 \mathrm{~dB}$ corresponds to a reflection efficiency of less than $9.85 \%(\mathrm{R}<0.1)$, in other words, over $90 \%$ of the incident EM waves will be absorbed. From Fig. 5d, the layered PVDF nanocomposites (30 wt $\%$ SiCnw, 0\% VF) exhibited an optimal low reflection bandwidth of $2.1 \mathrm{GHz}$, and the corresponding peak $\mathrm{SE}_{\mathrm{R}}$ obtained is $0.29 \mathrm{~dB}$ at an absorption layer thickness of $1.05 \mathrm{~mm}$. Such absorption performance is attributed to the excellent dipolar polarization loss of the incorporated SiCnw [35].

By inducing foaming in the absorption layer with a $45 \%$ VF, the layered foam/film PVDF nanocomposites exhibited enhanced EM wave absorption performance, as shown in Fig. 5e-h. At a foam thickness of $1.8 \mathrm{~mm}$, the layered foam/ film PVDF nanocomposite exhibited a broader optimal LR bandwidth from 2.1 to $2.4 \mathrm{GHz}$ and smaller corresponding minimal $\mathrm{SE}_{\mathrm{R}}$ from 0.36 to $0.011 \mathrm{~dB}$ compared to its solid counterpart. This value corresponds to only $0.25 \%$ reflection efficiency. The presence of cellular structure can help tailor the impedance match by reducing the real permittivity of the absorber, and fewer incident EM waves will be reflected at the surface [35, 41]. Meanwhile, the microstructures shown in Fig. 4 can provide abundant solid-air interfaces where the propagating EM wave undergoes internal scattering within each bubble [19, 34, 41, 78]. This prolongs the propagation path of EM waves in the foam. Eventually, the propagating EM wave will be further attenuated in the foamed absorption layer.

The absorption phase exhibited a severe impedance mismatch by further increasing the void content from 45 to $55 \%$. From Fig. 5i-1, the layered foam/film nanocomposite had a narrower optimal low reflection bandwidth of $1.7 \mathrm{GHz}$ with an optimal $\mathrm{SE}_{\mathrm{R}}$ of $0.38 \mathrm{~dB}$ at a foam thickness of $2.2 \mathrm{~mm}$. In this case, the poor impedance mismatch is attributed to insufficient absorption region and dissipation capability of the over-foamed absorber. This is also evident in Fig. S9a, where the AC conductivity of the PVDF/30 wt\% SiCnw composite foam $(65 \% \mathrm{VF})$ is reduced by nearly 1 order of magnitude at $1 \mathrm{kHz}$, from $1.9 \times 10^{-7}$ to $5.6 \times 10^{-8} \mathrm{~S} \mathrm{~m}^{-1}$, as compared to the layered foam/film nanocomposite with $45 \% \mathrm{VF}$.

By incorporating MXene nanosheets in the absorption layer, the layered foam/film PVDF nanocomposites (30 wt\% SiCnw@MXene7:1) exhibited enhanced EM wave attenuation capability, as shown in Figs. 6 and S10. With the same $45 \%$ VF (Fig. 6a-d), the layered foam/film PVDF nanocomposite (30 wt\% SiCnw@MXene 7:1) exhibited a broader optimal LR bandwidth of $3.1 \mathrm{GHz}$ with a peak $\mathrm{SE}_{\mathrm{R}}$ of $0.086 \mathrm{~dB}$ at a foam thickness of $1.45 \mathrm{~mm}$, as compared to the layered foam/film PVDF nanocomposites without MXene nanosheets incorporated (30 wt\% SiCnw). In this case, the broader low reflection bandwidth suggests that the heterostructures of SiCnw/MXene in the PVDF matrix can effectively tune the dissipation capability and impedance matching. Also, the foam thickness to achieve optimal low reflection bandwidth was reduced from 1.8 to $1.45 \mathrm{~mm}$. This indicates enhanced dielectric permittivity of the absorber, attributed to the intensified interfacial polarization because of the addition of MXene nanosheets. The conduction loss of the layered foam/film PVDF nanocomposites is also enhanced with incorporation of MXene nanosheets. As shown in Fig. S9a, c, two absorbers (30 wt\% SiCnw@ MXene7:1 and $30 \mathrm{wt} \%$ SiCnw, 65\% VF) had AC conductivity of $9.7 \times 10^{-7}$ and $1.6 \times 10^{-7} \mathrm{~S} \mathrm{~m}^{-1}$, respectively. The 

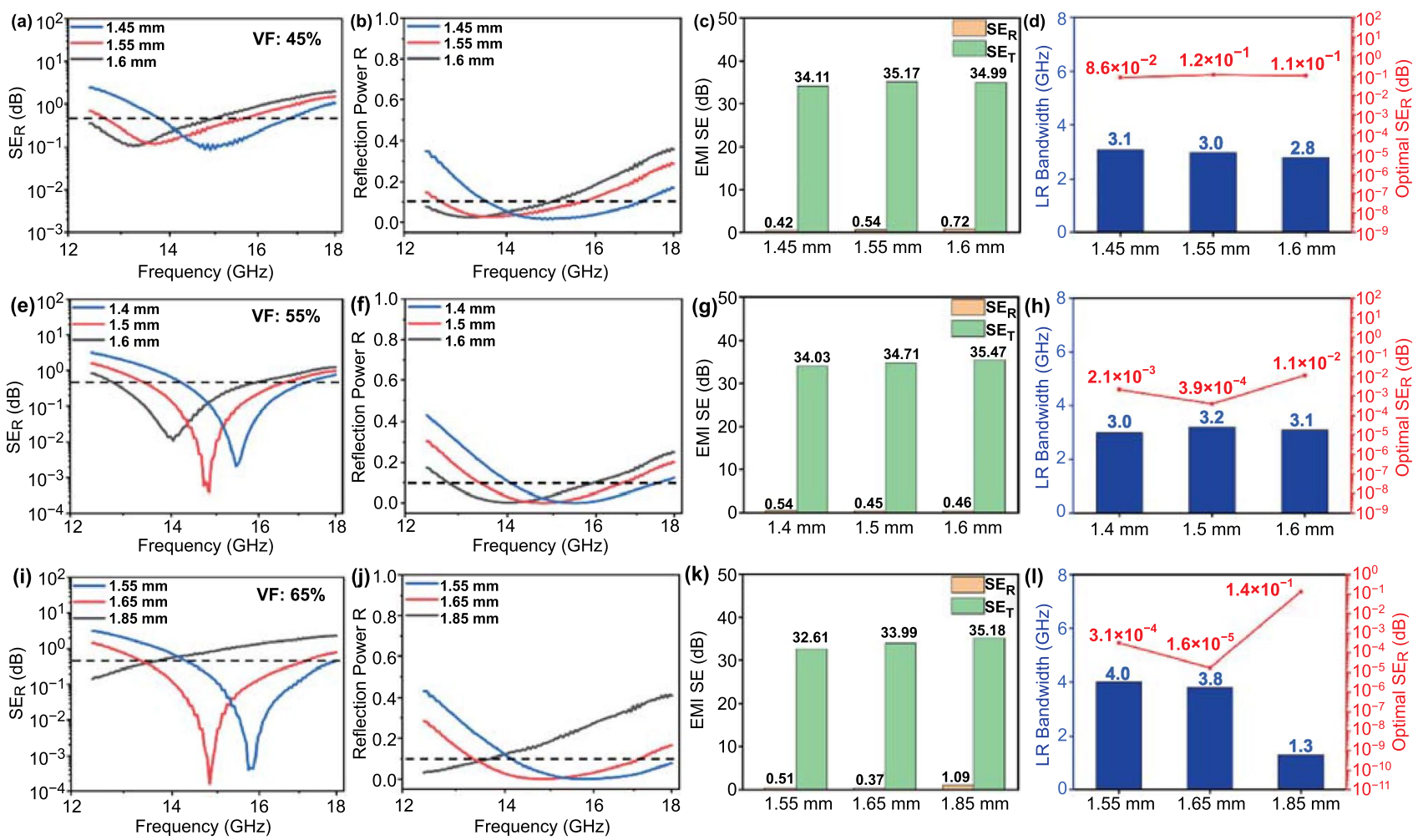

Fig. 6 EMI shielding performance of layered foam/film PVDF nanocomposites (30 wt\% SiCnw@MXene7:1) corresponds to different matching thicknesses of absorption layer; $\mathbf{a}, \mathbf{e}, \mathbf{i} \mathrm{SE}_{\mathrm{R}} ; \mathbf{b}, \mathbf{f}, \mathbf{j}$ reflection power $\mathrm{R} ; \mathbf{c}, \mathbf{g}, \mathbf{k}$ average value of $\mathrm{SE}_{\mathrm{R}}$ and $\mathrm{SE}_{\mathrm{T}} ; \mathbf{d}, \mathbf{h}, \mathbf{l}$ low reflection bandwidth and minimal $\mathrm{SE}_{\mathrm{R}}$ value. Dependence of the VF: a-d $45 \% \mathrm{VF}$; e-h $55 \% \mathrm{VF}$; and $\mathbf{i}-\mathrm{l} 65 \% \mathrm{VF}$

bottom reflection layer was removed by sanding when the ac conductivity of the upper absorption layer was measured.

By increasing the VF of absorber from 45 to $55 \%$ (Fig. 6e-h), the increased void content led to promoted impedance matching, where layered foam/film PVDF nanocomposite had a broader optimal low reflection bandwidth of $3.2 \mathrm{GHz}$, with a peak $\mathrm{SE}_{\mathrm{R}}$ of $3.9 \times 10^{-4} \mathrm{~dB}$ at a foam thickness of $1.5 \mathrm{~mm}$. Moreover, by further increasing the VF of absorber to $65 \%$ (Fig. 6i-1), the layered foam/film nanocomposite had a broader LR bandwidth of $4.0 \mathrm{GHz}$ with an optimal $\mathrm{SE}_{\mathrm{R}}$ of $3.1 \times 10^{-4} \mathrm{~dB}$ at a foam thickness of $1.55 \mathrm{~mm}$. This $\mathrm{SE}_{\mathrm{R}}$ value corresponds to only $0.0022 \%$ reflection efficiency. The density of the product should also be considered to evaluate the EMI shielding properties. At the optimal condition, SiCnw@MXene7:1 layered foam/film PVDF nanocomposite (65\% VF) had a $42 \%$ of reduction in area density from 0.322 to $0.187 \mathrm{~g} \mathrm{~cm}^{-2}$, as compared to its original ( $30 \mathrm{wt} \% \mathrm{SiCnw}$ ) solid counterpart (Table 1).

Increasing the VF can effectively tune the impedance matching and intensify internal scattering and multi-reflection, leading to a broader optimal low reflection bandwidth. However, excessively high void content can further break the embedded fillers apart around growing cells, leading to reduction in effective permittivity, conductivity and dissipation capability. The effect of the VF on the EMI shielding properties has been further explored in Fig. S11. The EM wave reflection performance for layered foam/film PVDF nanocomposites (30wt\% SiCnw@MXene9:1) with various VFs of the absorption layer at the same thickness has been investigated.

Moreover, with a higher MXene content, higher values of optimum VF can be achieved before compromising the absorption and dissipation capability. At a fixed VF of 55\%, by increasing the MXene content (30 wt\%, SiCnw:MXene from 1:0 to 9:1 and to 7:1), the $\mathrm{SE}_{\mathrm{A}}$ of composite foams increased from 2.6 to $3.75 \mathrm{~dB}$, and to $4.01 \mathrm{~dB}$, respectively (Fig. S12a).

The summary of the experimental results presented in Table 1, the layered foam/film PVDF nanocomposite (30wt\% SiCnw@MXene9:1) had an optimal low reflection bandwidth broadened from 3.2 to $3.9 \mathrm{~dB}$ as the VF 
Table 1 EMI shielding properties for layered foam/film PVDF nanocomposites with various filler compositions, VF and absorption layer thickness

\begin{tabular}{|c|c|c|c|c|c|c|}
\hline Absorption layer filler content & $\begin{array}{l}\text { Void frac- } \\
\text { tion } \%\end{array}$ & $\begin{array}{l}\text { Total thickness } \\
(\mathrm{mm})\end{array}$ & $\begin{array}{l}\text { Area density g } \\
\mathrm{cm}^{-2}\end{array}$ & Minimal $\mathrm{SE}_{R}(\mathrm{~dB})$ & $\begin{array}{l}\text { Average } \mathrm{SE}_{R} \\
\text { (dB) }\end{array}$ & $\begin{array}{l}\text { Bandwidth } \\
\mathrm{SE}_{R}<0.45 \mathrm{~dB} \\
(\mathrm{GHz})\end{array}$ \\
\hline \multirow[t]{3}{*}{$30 \mathrm{wt} \% \mathrm{SiCnw}$} & 0 & 1.6 & 0.322 & 0.36 & 0.72 & 1.9 \\
\hline & 45 & 2.2 & 0.284 & $1.11 \times 10^{-2}$ & 0.63 & 2.4 \\
\hline & 55 & 2.6 & 0.281 & 0.38 & 0.74 & 1.4 \\
\hline \multirow[t]{5}{*}{30 wt\% SiCnw@ MXene 9:1 } & 45 & 1.9 & 0.238 & $1.48 \times 10^{-2}$ & 0.46 & 3.2 \\
\hline & 55 & 2.4 & 0.259 & $2.48 \times 10^{-6}$ & 0.58 & 3.0 \\
\hline & 55 & 2.2 & 0.241 & $5.41 \times 10^{-4}$ & 0.39 & 3.2 \\
\hline & 55 & 2.0 & 0.223 & $2.08 \times 10^{-4}$ & 0.63 & 3.5 \\
\hline & 65 & 2.4 & 0.219 & $5.41 \times 10^{-2}$ & 0.43 & 3.9 \\
\hline \multirow[t]{4}{*}{30 wt\% SiCnw@ MXene 7:1 } & 45 & 1.85 & 0.239 & $8.61 \times 10^{-2}$ & 0.42 & 3.1 \\
\hline & 55 & 1.9 & 0.212 & $3.90 \times 10^{-4}$ & 0.45 & 3.2 \\
\hline & 65 & 2.05 & 0.194 & $1.65 \times 10^{-4}$ & 0.37 & 3.8 \\
\hline & 65 & 1.95 & 0.187 & $3.10 \times 10^{-4}$ & 0.51 & 4.0 \\
\hline
\end{tabular}

Reflection Layer $\mathbf{0 . 4}$ mm (PVDF/20 wt\% GnP@CNT)-Average $S E_{T} 33.49 \mathrm{~dB}$

increased from 45 to 65\%. The SiCnw@MXene9:1 layered foam/film PVDF nanocomposite (65\% VF) showed a similar optimal low reflection bandwidth $(\sim 4 \mathrm{GHz})$ to those of the SiCnw@MXene7:1 layered foam/film nanocomposite (65\% VF). However, the SiCnw@MXene9:1 layered foam/ film PVDF nanocomposite (65\% VF) had a higher matching thickness of $2.0 \mathrm{~mm}$ for optimal low reflection bandwidth. Also, the minimum $\mathrm{SE}_{\mathrm{R}}$ obtained is two orders of magnitude higher than that of the SiCnw@MXene7:1 layered foam/film PVDF nanocomposite (65\% VF). It is clear that the effective permittivity and dissipation capability of the SiCnw@MXene9:1 layered foam/film PVDF nanocomposite $(65 \% \mathrm{VF})$ are insufficient due to over-foaming, as compared to the SiCnw@MXene7:1 layered foam/film nanocomposite (65\% VF) (shown in Figs. S13 and S14). The EMI shielding properties of the absorption layers (without the reflection layer) are shown in Fig. S12a. The larger void fraction in absorption layers often leads to a lowered $\mathrm{SE}_{\mathrm{R}}$. For instance, PVDF SiCnw@MXene foam (30wt\% 9:1, $55 \% \mathrm{VF}, 1.8 \mathrm{~mm}$ ) obtained a $\mathrm{SE}_{\mathrm{R}}$ of $2.79 \mathrm{~dB}$, while PVDF SiCnw@MXene foam (30wt\% 7:1, 65\% VF, 1.8 mm) exhibited a $\mathrm{SE}_{\mathrm{R}}$ of $2.39 \mathrm{~dB}$ regardless of the increased MXene content. In the meantime, both composite foams obtained a $\mathrm{SE}_{\mathrm{A}}$ of around $3.75 \mathrm{~dB}$, indicating that foam $(7: 1,65 \%$ VF) obtained better impedance matching (lower reflectivity) while maintaining the high EM wave attenuation capability. Moreover, by increasing the MXene content (9:1 to 7:1), the $\mathrm{AC}$ conductivity of the absorption layer increased from $2.2 \times 10^{-7}$ to $4.5 \times 10^{-7} \mathrm{~S} \mathrm{~m}^{-1}$ at $1 \mathrm{kHz}$ (Fig. S9b, c). Detailed EMI shielding properties of layered foam/film PVDF nanocomposite (30wt\% SiCnw@ MXene9:1) can be found in Figs. S13 and S14.

On the other hand, the SiCnw@MXene9:1 layered foam/ film PVDF nanocomposite (45\% VF) exhibited a smaller peak $\mathrm{SE}_{\mathrm{R}}$ of $0.015 \mathrm{~dB}$ and a broader optimal low reflection bandwidth of $3.2 \mathrm{~dB}$ at a foam thickness of $1.5 \mathrm{~mm}$, as compared to those of the SiCnw@MXene7:1 layered foam/film PVDF nanocomposite (45\% VF). With higher MXene content, the SiCnw@MXene7:1 layered foam/film PVDF nanocomposites exhibit higher impedance mismatch and reflection at 45\% VF compared to those of SiCnw@ MXene9:1 layered foam/film PVDF nanocomposite. This indicates that a higher VF is required to achieve optimal impedance matching of the absorber with higher MXene content.

As shown in Table S1, the foamability of PVDF/30 wt $\%$ SiCnw@MXene5:1 nanocomposite is significantly restricted due to the addition of MXene. A maximum VF of about 35\% was obtained, which is insufficient to tune the impedance matching of the absorption phase (Fig. S9d).

Figure S15 illustrated some of the recent advances made in the development of EMI shielding materials [16, $30,40,46,79-84]$, which are compared with the overall EMI shielding effectiveness and reflection of the layered foam/film PVDF nanocomposites reported in this study. 
The superior electrical conductivity is often prerequisite for achieving high EMI SE in the traditional EMI shielding materials. However, high electrical conductivity will also lead to a severe impedance mismatch and high reflectivity. As shown in Fig. S14, compared with other EMI shielding composites, the layered foam/film PVDF nanocomposite (30 wt\% SiCnw@MXene 7:1,65\% VF) can achieve the average reflectivity $(\mathrm{R})$ as low as 0.08 while maintaining efficient shielding effectiveness of $32.48 \mathrm{~dB}$ highlighting the supremacy of this work.

The effective EMI shielding with ultra-low reflection efficiency of layered foam/film PVDF nanocomposites developed in this study can be attributed to the dissipation mechanisms presented in Fig. 7. The EM waves can penetrate the absorption layer and its microcellular structure because of good impedance matching. The penetrated waves will be dissipated by the numerous heterogeneous interfaces between SiCnw, MXene nanosheets and PVDF matrix via interfacial polarization loss, dipolar polarization loss and conduction loss. Furthermore, abundant solid-air interfaces obtained from the microcellular structure can trap the penetrated EM wave via scattering and multiple reflections. This prolongs the trajectory of the EM wave propagation within foams leading to further attenuation. The remaining propagating EM waves will be mostly reflected by the highly conductive reflection layer of PVDF/MWCNT/GnPs composite film and propagate through the absorption layer again. Finally, EM wave energy will be consumed by the interference between the second reflected wave and surface reflected EM wave, based on the law of quarter wavelength cancellation. In general, the layered foam/film PVDF nanocomposites achieved both efficient EMI SE and low reflectivity through (i) enhanced surface impedance matching of the foamed upper absorption phase, (ii) excellent EM wave attenuation via enhanced dielectric loss offered by the embedded hybrid heterostructures of SiCnw@MXene in the absorption layer, (iii) the efficient shielding effectiveness of the reflection layer achieved as a result of superior electrical conductivity and high reflection capability, and (iv) triggering quarter wavelength cancellation of incident EM wave by tuning the thickness of absorption phase.

\section{Summary and Conclusions}

Layered foam/film PVDF nanocomposites with high-efficiency EMI SE and ultra-low reflection characteristics are introduced. The layered nanocomposites were composed of PVDF/SiCnw@MXene composite foam as the upper absorption layer and PVDF/MWCNT/GnPs composite film as the bottom reflection layer. The layered foam/film structure was

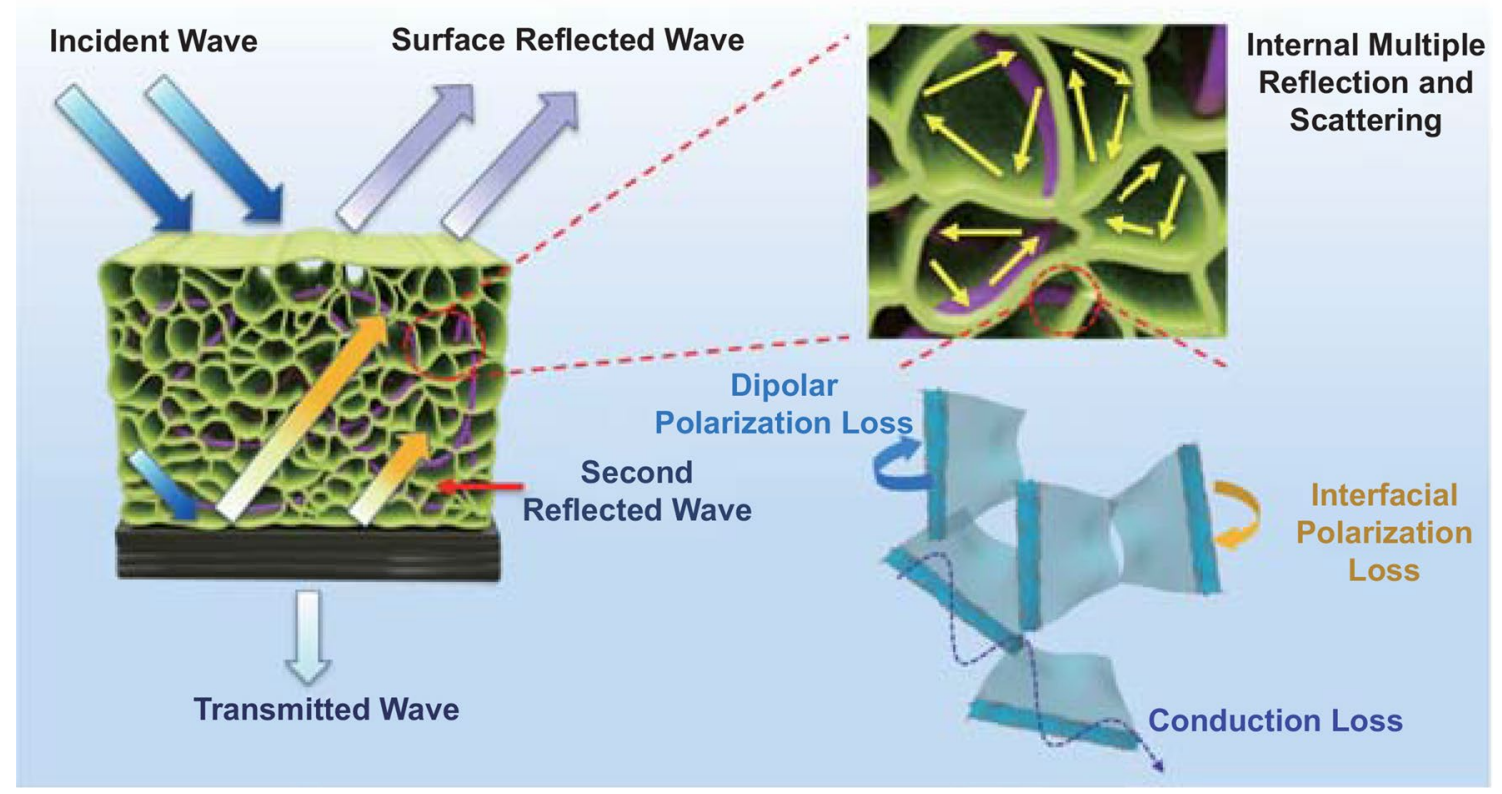

Fig. 7 Schematic illustration of EM wave dissipation in the layered foam/film PVDF nanocomposites 
developed by leveraging the different crystal melting temperatures of two grades of PVDF in the batch foaming process. The absorption layer's impedance matching and dissipation capability were effectively tuned by developing heterogeneous interfaces between SiCnw, MXene nanosheets and PVDF matrix, and by introducing microcellular structure. Meanwhile, the 0.4-mm-thick highly conductive PVDF/10 wt $\%$ MWCNT/10 wt\% GnPs composite film performed as an excellent reflection layer. The synergy between absorption and reflection layers, along with optimized filler content/ratio and VF of the EM wave shielding and attenuation properties of the nanocomposites, was maximized. For instance, the layered foam/film PVDF nanocomposite (30 wt\% SiCnw@MXene 7:1,65\% VF) exhibited an average EMI SE of $32.6 \mathrm{~dB}$ with low reflection bandwidth of $4 \mathrm{GHz}$ and minimal $\mathrm{SE}_{\mathrm{R}}$ of $3.1 \times 10^{-4} \mathrm{~dB}$ over the Ku-band (12.4-18 GHz). The design of layered foam/film structure in this work presents a novel and simple strategy for developing high-efficiency, lightweight and wideband low reflection EMI shielding materials for cutting edge applications.

\section{Supporting Information}

The SEM images of fabricated composites, XPS results, AC electrical conductivity and EMI shielding properties.

Acknowledgements The authors gratefully acknowledge and appreciate the financial support of NSERC (Discovery Grant RGPIN-2015-03985). The authors would like to acknowledge material donation from Arkema in the USA.

Funding Open access funding provided by Shanghai Jiao Tong University.

Open Access This article is licensed under a Creative Commons Attribution 4.0 International License, which permits use, sharing, adaptation, distribution and reproduction in any medium or format, as long as you give appropriate credit to the original author(s) and the source, provide a link to the Creative Commons licence, and indicate if changes were made. The images or other third party material in this article are included in the article's Creative Commons licence, unless indicated otherwise in a credit line to the material. If material is not included in the article's Creative Commons licence and your intended use is not permitted by statutory regulation or exceeds the permitted use, you will need to obtain permission directly from the copyright holder. To view a copy of this licence, visit http://creativecommons.org/licenses/by/4.0/.
Supplementary Information The online version contains supplementary material available at https://doi.org/10.1007/ s40820-021-00759-4.

\section{References}

1. Who is Going to Use the $12 \mathrm{GHz}$ Band - 5G, Starlink, Both? I IE. (n.d.). Retrieved March 26, 2021 from https://interestin gengineering.com/who-is-going-to-use-the-12-ghz-band-5gstarlink-or-both

2. Military Radar Market to 2020 - X \& KU Band Radars are Expected to Grow Fastest in the Military Radars Market. (n.d.). Retrieved March 26, 2021 from https://www.prnew swire.com/news-releases/military-radar-market-to-2020--$\mathrm{x}$--ku-band-radars-are-expected-to-grow-fastest-in-the-milit ary-radars-market-570914721.html

3. P.M. Mariappan, D.R. Raghavan, S.H.E. Abdel Aleem, A.F. Zobaa, Effects of electromagnetic interference on the functional usage of medical equipment by $2 \mathrm{G} / 3 \mathrm{G} / 4 \mathrm{G}$ cellular phones: a review. J. Adv. Res. 7, 727-738 (2016). https://doi. org/10.1016/j.jare.2016.04.004

4. G.M. Weng, J. Li, M. Alhabeb, C. Karpovich, H. Wang et al., Layer-by-layer assembly of cross-functional semi-transparent MXene-carbon nanotubes composite films for next-generation electromagnetic interference shielding. Adv. Funct. Mater. 28, 1803360 (2018). https://doi.org/10.1002/adfm.201803360

5. Z. Chen, C. Xu, C. Ma, W. Ren, H.M. Cheng, Lightweight and flexible graphene foam composites for high-performance electromagnetic interference shielding. Adv. Mater. 25, 1296-1300 (2013). https://doi.org/10.1002/adma.201204196

6. J. Xu, L. Xia, J. Luo, S. Lu, X. Huang et al., High-performance electromagnetic wave absorbing CNT/SiCf composites: synthesis, tuning, and mechanism. ACS Appl. Mater. Interfaces 12, 20775-20784 (2020). https://doi.org/10.1021/acsami. 9b19281

7. W.T. Cao, F.F. Chen, Y.J. Zhu, Y.G. Zhang, Y.Y. Jiang et al., Binary strengthening and toughening of MXene/cellulose nanofiber composite paper with nacre-inspired structure and superior electromagnetic interference shielding properties. ACS Nano 12, 4583-4593 (2018). https://doi.org/10.1021/ acsnano.8b00997

8. M.S. Cao, X.X. Wang, M. Zhang, J.C. Shu, W.Q. Cao et al., Electromagnetic response and energy conversion for functions and devices in low-dimensional materials. Adv. Funct. Mater. 29, 1807398 (2019). https://doi.org/10.1002/adfm.201807398

9. H. Xu, X. Yin, X. Li, M. Li, S. Liang et al., Lightweight $\mathrm{Ti}_{2} \mathrm{CT}_{\mathrm{x}} \mathrm{MXene/poly}$ (vinyl alcohol) composite foams for electromagnetic wave shielding with absorption-dominated feature. ACS Appl. Mater. Interfaces 11, 10198-10207 (2019). https://doi.org/10.1021/acsami.8b21671 
10. B. Du, M. Cai, X. Wang, J. Qian, C. He et al., Enhanced electromagnetic wave absorption property of binary $\mathrm{ZnO} / \mathrm{NiCo}_{2} \mathrm{O}_{4}$ composites. J. Adv. Ceram. 10, 832-842 (2021). https://doi. org/10.1007/s40145-021-0476-Z

11. W. Zhang, B. Zhao, H. Xiang, F.Z. Dai, S. Wu et al., One-step synthesis and electromagnetic absorption properties of high entropy rare earth hexaborides (HE REB6) and high entropy rare earth hexaborides/borates (HE REB6/HE REBO3) composite powders. J. Adv. Ceram. 10, 62-77 (2021). https://doi. org/10.1007/s40145-020-0417-2

12. X. Ye, Z. Chen, S. Ai, B. Hou, J. Zhang et al., Porous SiC/ melamine-derived carbon foam frameworks with excellent electromagnetic wave absorbing capacity. J. Adv. Ceram. 8, 479-488 (2019). https://doi.org/10.1007/s40145-019-0328-2

13. H. Pang, L. Xu, D.X. Yan, Z.M. Li, Conductive polymer composites with segregated structures. Prog. Polym. Sci. 39, 1908-1933 (2014). https://doi.org/10.1016/j.progpolymsci. 2014.07.007

14. P. He, M.-S. Cao, W.-Q. Cao, J. Yuan, Developing MXenes from wireless communication to electromagnetic attenuation. Nano-Micro Lett. 13, 131 (2021). https://doi.org/10.1007/ S40820-021-00645-Z

15. F. Shahzad, M. Alhabeb, C.B. Hatter, B. Anasori, S.M. Hong et al., Electromagnetic interference shielding with 2D transition metal carbides (MXenes). Science 353, 1137-1140 (2016). https://doi.org/10.1126/science.aag2421

16. A. Iqbal, F. Shahzad, K. Hantanasirisakul, M.-K. Kim, J. Kwon, J. Hong, H. Kim, D. Kim, Y. Gogotsi, and C. Min Koo, Anomalous Absorption of Electromagnetic Waves by $2 D$ Transition Metal Carbonitride $\mathrm{Ti}_{3} \mathrm{CNT}_{x}$ (MXene) (American Association for the Advancement of Science, 2020). https:// doi.org/10.1126/science.aba7977

17. B. Zhao, C. Zhao, M. Hamidinejad, C. Wang, R. Li et al., Incorporating a microcellular structure into PVDF/graphenenanoplatelet composites to tune their electrical conductivity and electromagnetic interference shielding properties. J. Mater. Chem. C 6, 10292-10300 (2018). https://doi.org/10. 1039/C8TC03714K

18. B. Zhao, S. Wang, C. Zhao, R. Li, S.M. Hamidinejad et al., Synergism between carbon materials and Ni chains in flexible poly(vinylidene fluoride) composite films with high heat dissipation to improve electromagnetic shielding properties. Carbon 127, 469-478 (2018). https://doi.org/10.1016/j.carbon. 2017.11.032

19. M. Hamidinejad, B. Zhao, A. Zandieh, N. Moghimian, T. Filleter et al., Enhanced electrical and electromagnetic interference shielding properties of polymer-graphene nanoplatelet composites fabricated via supercritical-fluid treatment and physical foaming. ACS Appl. Mater. Interfaces 10, 3075230761 (2018). https://doi.org/10.1021/acsami.8b10745

20. M. Hamidinejad, A. Zandieh, J.H. Lee, J. Papillon, B. Zhao et al., Insight into the directional thermal transport of hexagonal boron nitride composites. ACS Appl. Mater. Interfaces 11, 41726-41735 (2019). https://doi.org/10.1021/acsami.9b16070
21. C. Liang, M. Hamidinejad, L. Ma, Z. Wang, C.B. Park, Lightweight and flexible graphene/SiC-nanowires/ poly(vinylidene fluoride) composites for electromagnetic interference shielding and thermal management. Carbon 156, 58-66 (2020). https://doi.org/10.1016/j.carbon.2019.09.044

22. L. Liang, R. Yang, G. Han, Y. Feng, B. Zhao et al., Enhanced electromagnetic wave-absorbing performance of magnetic nanoparticles-anchored 2D $\mathrm{Ti}_{2} \mathrm{CT}_{\mathrm{x}}$ MXene. ACS Appl. Mater. Interfaces 12, 2644-2654 (2020). https://doi.org/10.1021/ acsami.9b18504

23. C. Liang, Z. Wang, Controllable fabricating dielectric-dielectric SiC@C core-shell nanowires for high-performance electromagnetic wave attenuation. ACS Appl. Mater. Interfaces 9, 40690-40696 (2017). https://doi.org/10.1021/acsami.7b13063

24. J. Wang, Z. Jia, X. Liu, J. Dou, B. Xu et al., Construction of 1D heterostructure NiCo@C/ZnO nanorod with enhanced microwave absorption. Nano-Micro Lett. 13, 175 (2021). https://doi. org/10.1007/S40820-021-00704-5

25. F. Pan, Z. Liu, B. Deng, Y. Dong, X. Zhu et al., Lotus leafderived gradient hierarchical porous $\mathrm{C} / \mathrm{MoS}_{2}$ morphology genetic composites with wideband and tunable electromagnetic absorption performance. Nano-Micro Lett. 13, 43 (2021). https://doi.org/10.1007/S40820-020-00568-1

26. G. He, Y. Duan, H. Pang, Microwave absorption of crystalline $\mathrm{Fe} / \mathrm{MnO} @ \mathrm{C}$ nanocapsules embedded in amorphous carbon. Nano-Micro Lett. 12, 57 (2020). https://doi.org/10.1007/ s40820-020-0388-4

27. R. Che, L.M. Peng, X. Duan, Q. Chen, X. Liang, Microwave absorption enhancement and complex permittivity and permeability of Fe encapsulated within carbon nanotubes. Adv. Mater. 16, 401-405 (2004). https://doi.org/10.1002/adma. 200306460

28. B. Zhao, C. Zhao, R. Li, S.M. Hamidinejad, C.B. Park, Flexible, ultrathin, and high-efficiency electromagnetic shielding properties of poly(vinylidene fluoride)/carbon composite films. ACS Appl. Mater. Interfaces 9, 20873-20884 (2017). https://doi.org/10.1021/acsami.7b04935

29. L. Liang, G. Han, Y. Li, B. Zhao, B. Zhou et al., Promising $\mathrm{Ti}_{3} \mathrm{C}_{2} \mathrm{~T}_{\mathrm{x}} \mathrm{MXene} / \mathrm{Ni}$ chain hybrid with excellent electromagnetic wave absorption and shielding capacity. ACS Appl. Mater. Interfaces 11, 25399-25409 (2019). https://doi.org/10. 1021/acsami.9b07294

30. P. Song, B. Liu, C. Liang, K. Ruan, H. Qiu et al., Lightweight, flexible cellulose-derived carbon aerogel@reduced graphene oxide/PDMS composites with outstanding EMI shielding performances and excellent thermal conductivities. Nano-Micro Lett. 13, 3 (2021). https://doi.org/10.1007/ s40820-021-00624-4

31. Y. Chen, H. Bin Zhang, Y. Yang, M. Wang, A. Cao et al., High-performance epoxy nanocomposites reinforced with three-dimensional carbon nanotube sponge for electromagnetic interference shielding. Adv. Funct. Mater. 26, 447-455 (2016). https://doi.org/10.1002/adfm.201503782

32. Z. Wang, R. Wei, J. Gu, H. Liu, C. Liu et al., Ultralight, highly compressible and fire-retardant graphene aerogel with 
self-adjustable electromagnetic wave absorption. Carbon 139, 1126-1135 (2018). https://doi.org/10.1016/j.carbon.2018.08. 014

33. H. Zhao, Y. Cheng, W. Liu, L. Yang, B. Zhang et al., Biomassderived porous carbon-based nanostructures for microwave absorption. Nano-Micro Lett. 11, 24 (2019). https://doi.org/ 10.1007/s40820-019-0255-3

34. Z. Zeng, H. Jin, M. Chen, W. Li, L. Zhou et al., Lightweight and anisotropic porous MWCNT/WPU composites for ultrahigh performance electromagnetic interference shielding. Adv. Funct. Mater. 26, 303-310 (2016). https://doi.org/10.1002/ adfm.201503579

35. C. Liang, Z. Wang, L. Wu, X. Zhang, H. Wang et al., Light and strong hierarchical porous $\mathrm{SiC}$ foam for efficient electromagnetic interference shielding and thermal insulation at elevated temperatures. ACS Appl. Mater. Interfaces 9, 29950-29957 (2017). https://doi.org/10.1021/acsami.7b07735

36. W.L. Song, M.S. Cao, L.Z. Fan, M.M. Lu, Y. Li et al., Highly ordered porous carbon/wax composites for effective electromagnetic attenuation and shielding. Carbon 77, 130-142 (2014). https://doi.org/10.1016/j.carbon.2014.05.014

37. B. Zhao, M. Hamidinejad, S. Wang, P. Bai, R. Che et al., Advances in electromagnetic shielding properties of composite foams. J. Mater. Chem. A 9, 8896-8949 (2021). https://doi. org/10.1039/d1ta00417d

38. A. Ameli, P.U. Jung, C.B. Park, Electrical properties and electromagnetic interference shielding effectiveness of polypropylene/carbon fiber composite foams. Carbon 60, 379-391 (2013). https://doi.org/10.1016/j.carbon.2013.04.050

39. A. Ameli, M. Nofar, S. Wang, C.B. Park, Lightweight polypropylene/stainless-steel fiber composite foams with low percolation for efficient electromagnetic interference shielding. ACS Appl. Mater. Interfaces 6, 11091-11100 (2014). https://doi. org/10.1021/am500445g

40. R. Li, L. Ding, Q. Gao, H. Zhang, D. Zeng et al., Tuning of anisotropic electrical conductivity and enhancement of EMI shielding of polymer composite foam via $\mathrm{CO}_{2}$-assisted delamination and orientation of MXene. Chem. Eng. J. 415, 128930 (2021). https://doi.org/10.1016/j.cej.2021.128930

41. B. Zhao, J. Deng, C. Zhao, C. Wang, Y.G. Chen et al., Achieving wideband microwave absorption properties in PVDF nanocomposite foams with an ultra-low MWCNT content by introducing a microcellular structure. J. Mater. Chem. C 8, 58-70 (2020). https://doi.org/10.1039/c9tc04575a

42. B. Zhao, R. Wang, Y. Li, Y. Ren, X. Li et al., Dependence of electromagnetic interference shielding ability of conductive polymer composite foams with hydrophobic properties on cellular structure. J. Mater. Chem. C 8, 7401-7410 (2020). https://doi.org/10.1039/d0tc00987c

43. S. Wang, Y. Huang, E. Chang, C. Zhao, A. Ameli et al., Evaluation and modeling of electrical conductivity in conductive polymer nanocomposite foams with multiwalled carbon nanotube networks. Chem. Eng. J. (2021). https://doi.org/10.1016/j. cej.2020.128382

44. S. Wang, Y. Huang, C. Zhao, E. Chang, A. Ameli et al., Theoretical modeling and experimental verification of percolation threshold with MWCNTs' rotation and translation around a growing bubble in conductive polymer composite foams. Compos. Sci. Technol. 199, 108345 (2020). https://doi.org/ 10.1016/j.compscitech.2020.108345

45. H. Duan, H. Zhu, J. Gao, D.X. Yan, K. Dai et al., Asymmetric conductive polymer composite foam for absorption dominated ultra-efficient electromagnetic interference shielding with extremely low reflection characteristics. J. Mater. Chem. A 8, 9146-9159 (2020). https://doi.org/10.1039/d0ta01393e

46. J. Yang, X. Liao, G. Wang, J. Chen, W. Tang et al., Fabrication of lightweight and flexible silicon rubber foams with ultraefficient electromagnetic interference shielding and adjustable low reflectivity. J. Mater. Chem. C 8, 147-157 (2019). https:// doi.org/10.1039/c9tc05152j

47. J. Yang, X. Liao, G. Wang, J. Chen, P. Song et al., Heterogeneous silicon rubber composite foam with gradient porous structure for highly absorbed ultra-efficient electromagnetic interference shielding. Compos. Sci. Technol. 206, 108663 (2021). https://doi.org/10.1016/j.compscitech.2021.108663

48. L. Ma, M. Hamidinejad, C. Liang, B. Zhao, S. Habibpour et al., Enhanced electromagnetic wave absorption performance of polymer/SiC-nanowire/MXene $\left(\mathrm{Ti}_{3} \mathrm{C}_{2} \mathrm{~T}_{\mathrm{x}}\right)$ composites. Carbon 179, 408-416 (2021). https://doi.org/10.1016/j.carbon. 2021.04.063

49. Q. Qi, L. Ma, B. Zhao, S. Wang, X. Liu et al., An effective design strategy for the sandwich structure of PVDF/GNP-NiCNT composites with remarkable electromagnetic interference shielding effectiveness. ACS Appl. Mater. Interfaces 12, 36568-36577 (2020). https://doi.org/10.1021/acsami.0c10600

50. X. Li, X. Yin, H. Xu, M. Han, M. Li et al., Ultralight MXenecoated, interconnected SiCnws three-dimensional lamellar foams for efficient microwave absorption in the X-band. ACS Appl. Mater. Interfaces 10, 34524-34533 (2018). https://doi. org/10.1021/acsami.8b13658

51. M. Naguib, O. Mashtalir, J. Carle, V. Presser, J. Lu et al., Two-dimensional transition metal carbides. ACS Nano 6, 1322-1331 (2012). https://doi.org/10.1021/nn204153h

52. M. Alhabeb, K. Maleski, B. Anasori, P. Lelyukh, L. Clark et al., Guidelines for synthesis and processing of two-dimensional titanium carbide $\left(\mathrm{Ti}_{3} \mathrm{C}_{2} \mathrm{~T}_{\mathrm{x}} \mathrm{MXene}\right)$. Chem. Mater. 29, 7633-7644 (2017). https://doi.org/10.1021/acs.chemmater. $7 \mathrm{~b} 02847$

53. H.P. Iwata, U. Lindefelt, S. Öberg, P.R. Briddon, Stacking faults in silicon carbide. in Physica B: Condensed Matter (North-Holland, 2003), pp. 165-170. https://doi.org/10.1016/j. physb.2003.09.045

54. C. Liang, C. Liu, H. Wang, L. Wu, Z. Jiang et al., SiC-Fe $\mathrm{O}_{4}$ dielectric-magnetic hybrid nanowires: Controllable fabrication, characterization and electromagnetic wave absorption. J. Mater. Chem. A 2, 16397-16402 (2014). https://doi.org/10. 1039/c4ta02907k

55. S.N. Leung, A. Wong, L.C. Wang, C.B. Park, Mechanism of extensional stress-induced cell formation in polymeric foaming processes with the presence of nucleating agents. J. Supercrit. Fluids 63, 187-198 (2012). https://doi.org/10.1016/j.supflu.2011.12.018 
56. P. Martins, A.C. Lopes, S. Lanceros-Mendez, Electroactive phases of poly(vinylidene fluoride): determination, processing and applications. Prog. Polym. Sci. 39, 683-706 (2014). https://doi.org/10.1016/j.progpolymsci.2013.07.006

57. M. Mackey, L. Flandin, A. Hiltner, E. Baer, Confined crystallization of PVDF and a PVDF-TFE copolymer in nanolayered films. J. Polym. Sci. Part B Polym. Phys. 49, 1750-1761 (2011). https://doi.org/10.1002/polb.22375

58. C. Wang, S.N. Leung, M. Bussmann, W.T. Zhai, C.B. Park, Numerical investigation of nucleating-agent-enhanced heterogeneous nucleation. Ind. Eng. Chem. Res. 49, 12783-12792 (2010). https://doi.org/10.1021/ie1017207

59. M. Hamidinejad, R. Chu, B. Zhao, C.B. Park, T. Filleter, Enhanced thermal conductivity of graphene Nanoplateletpolymer nanocomposites fabricated via supercritical fluid assisted in-situ exfoliation. ACS Appl. Mater. Interfaces 10, 1225-1236 (2018). https://doi.org/10.1021/acsami.7b15170

60. X. Wu, T. Tu, Y. Dai, P. Tang, Y. Zhang et al., Direct ink writing of highly conductive MXene frames for tunable electromagnetic interference shielding and electromagnetic waveinduced Thermochromism. Nano-Micro Lett. 13, 1-15 (2021). https://doi.org/10.1007/S40820-021-00665-9

61. H.S. Faruque, C. Lacabanne, H.S. Faruquet, Anelastic and dielectric properties of polyether-polyamide copolymer PEBAX studied by a thermally stimulated depolarisation current method. J. Phys. D-Appl. Phys 20, 939-944 (1987). https:// doi.org/10.1088/0022-3727/20/7/017

62. M. Zhang, C. Han, W.-Q. Cao, M.-S. Cao, H.-J. Yang et al., A nano-micro engineering nanofiber for electromagnetic absorber, green shielding and sensor. Nano Lett. 13, 27 (2020). https://doi.org/10.1007/S40820-020-00552-9

63. M. Arjmand, M. Mahmoodi, S. Park, U. Sundararaj, An innovative method to reduce the energy loss of conductive filler/ polymer composites for charge storage applications. Compos. Sci. Technol. 78, 24-29 (2013). https://doi.org/10.1016/j. compscitech.2013.01.019

64. C.W. Nan, Y. Shen, J. Ma, Physical properties of composites near percolation. Annu. Rev. Mater. Res. 40, 131-151 (2010). https://doi.org/10.1146/annurev-matsci-070909-104529

65. J. Yuan, S. Yao, and P. Poulin, Dielectric constant of polymer composites and the routes to high-k or low-k nanocomposite materials. in Polymer Nanocomposites: Electrical and Thermal Properties (Springer International Publishing, Cham, 2016), pp. 3-28.

66. M. Mahmoodi, M. Arjmand, U. Sundararaj, S. Park, The electrical conductivity and electromagnetic interference shielding of injection molded multi-walled carbon nanotube/polystyrene composites. Carbon 50, 1455-1464 (2012). https://doi.org/10. 1016/j.carbon.2011.11.004

67. A. Ameli, S. Wang, Y. Kazemi, C.B. Park, P. Pötschke, A facile method to increase the charge storage capability of polymer nanocomposites. Nano Energy 15, 54-65 (2015). https://doi. org/10.1016/j.nanoen.2015.04.004

68. J. Ding, L. Wang, Y. Zhao, L. Xing, X. Yu et al., Boosted interfacial polarization from multishell $\mathrm{TiO}_{2} @ \mathrm{Fe}_{3} \mathrm{O}_{4} @ \mathrm{PPy}$ heterojunction for enhanced microwave absorption. Small 15, 1902885 (2019). https://doi.org/10.1002/smll.201902885

69. W.-L. Song, C. Gong, H. Li, X.-D. Cheng, M. Chen et al., Graphene-based sandwich structures for frequency selectable electromagnetic shielding. ACS Appl. Mater. Interfaces 9, 36119-36129 (2017). https://doi.org/10.1021/ACSAMI. 7B08229

70. W.C. Yu, G.Q. Zhang, Y.H. Liu, L. Xu, D.X. Yan et al., Selective electromagnetic interference shielding performance and superior mechanical strength of conductive polymer composites with oriented segregated conductive networks. Chem. Eng. J. 373, 556-564 (2019). https://doi.org/10.1016/J.CEJ. 2019.05.074

71. R. Gregorio, Determination of the $\alpha, \beta$, and $\gamma$ crystalline phases of poly(vinylidene fluoride) films prepared at different conditions. J. Appl. Polym. Sci. 100, 3272-3279 (2006). https://doi.org/10.1002/app.23137

72. X. Cai, T. Lei, D. Sun, L. Lin, A critical analysis of the $\alpha, \beta$ and $\gamma$ phases in poly(vinylidene fluoride) using FTIR. RSC Adv. 7, 15382-15389 (2017). https://doi.org/10.1039/c7ra0 $1267 \mathrm{e}$

73. J.E. Lee, S.N. Leung, Multi-stage crystallization mechanism of electroactive phase polyvinylidene fluoride induced by thermal and supercritical carbon dioxide processing. CrystEngComm 20, 4080-4089 (2018). https://doi.org/10.1039/c8ce00531a

74. L. Yan, C. Hong, B. Sun, G. Zhao, Y. Cheng et al., In situ growth of core-sheath heterostructural $\mathrm{SiC}$ nanowire arrays on carbon fibers and enhanced electromagnetic wave absorption performance. ACS Appl. Mater. Interfaces 9, 6320-6331 (2017). https://doi.org/10.1021/acsami.6b15795

75. Y. Li, X. Liu, X. Nie, W. Yang, Y. Wang et al., Multifunctional organic-inorganic hybrid aerogel for self-cleaning, heat-insulating, and highly efficient microwave absorbing material. Adv. Funct. Mater. 29, 1-9 (2019). https://doi.org/10.1002/adfm. 201807624

76. J. Ren, A. Zhang, X. Wang, The Recent Progress of MXenebased microwave absorption materials. Carbon (2020). https:// doi.org/10.1016/j.carbon.2020.12.060

77. Q. Liu, Q. Cao, H. Bi, C. Liang, K. Yuan et al., CoNi@ $\mathrm{SiO}_{2} @$ $\mathrm{TiO}_{2}$ and $\mathrm{CoNi} @ \mathrm{Air} @ \mathrm{TiO}_{2}$ microspheres with strong wideband microwave absorption. Adv. Mater. 28, 486-490 (2016). https://doi.org/10.1002/adma.201503149

78. B. Zhao, G. Shao, B. Fan, W. Zhao, Y. Xie et al., Synthesis of flower-like $\mathrm{CuS}$ hollow microspheres based on nanoflakes self-assembly and their microwave absorption properties. J. Mater. Chem. A 3, 10345-10352 (2015). https://doi.org/10. 1039/C5TA00086F

79. G. Sang, P. Xu, T. Yan, V. Murugadoss, N. Naik et al., Interface engineered microcellular magnetic conductive polyurethane nanocomposite foams for electromagnetic interference shielding. Nano-Micro Lett. 13, 153 (2021). https://doi.org/ 10.1007/s40820-021-00677-5

80. H. Liu, Y. Xu, J.P. Cao, D. Han, Q. Yang et al., Skin structured silver/three-dimensional graphene/polydimethylsiloxane composites with exceptional electromagnetic interference 
shielding effectiveness. Compos. Part A Appl. Sci. Manuf. 148, 106476 (2021). https://doi.org/10.1016/j.compositesa. 2021.106476

81. E. Kim, H. Zhang, J.H. Lee, H. Chen, H. Zhang et al., MXene/ polyurethane auxetic composite foam for electromagnetic interference shielding and impact attenuation. Compos. Part A Appl. Sci. Manuf. 147, 106430 (2021). https://doi.org/10. 1016/j.compositesa.2021.106430

82. H. Zhang, G. Zhang, Q. Gao, M. Tang, Z. Ma et al., Multifunctional microcellular PVDF/Ni-chains composite foams with enhanced electromagnetic interference shielding and superior thermal insulation performance. Chem. Eng. J. 379, 122304 (2020). https://doi.org/10.1016/j.cej.2019.122304

83. L. Kong, X. Yin, H. Xu, X. Yuan, T. Wang et al., Powerful absorbing and lightweight electromagnetic shielding CNTs/ RGO composite. Carbon 145, 61-66 (2019). https://doi.org/ 10.1016/J.CARBON.2019.01.009

84. T. Wang, W.-W. Kong, W.-C. Yu, J.-F. Gao, K. Dai et al., A healable and mechanically enhanced composite with segregated conductive network structure for high-efficient electromagnetic interference shielding. Nano-Micro Lett. 13, 162 (2021). https://doi.org/10.1007/S40820-021-00693-5 